\title{
Certified In-lined Reference Monitoring on .NET*
}

\author{
Kevin W. Hamlen \\ Cornell University
}

\author{
Greg Morrisett \\ Harvard University
}

Fred B. Schneider

Cornell University

November 16, 2005

\begin{abstract}
Mobile is an extension of the .NET Common Intermediate Language that supports certified In-Lined Reference Monitoring. Mobile programs have the useful property that if they are well-typed with respect to a declared security policy, then they are guaranteed not to violate that security policy when executed. Thus, when an In-Lined Reference Monitor (IRM) is expressed in Mobile, it can be certified by a simple type-checker to eliminate the need to trust the producer of the IRM.

Security policies in Mobile are declarative, can involve unbounded collections of objects allocated at runtime, and can regard infinitelength histories of security events exhibited by those objects. Our prototype implementation of Mobile enforces properties expressed by finite-state security automata - one automaton for each securityrelevant object, and can type-check Mobile programs in the presence of exceptions, finalizers, concurrency, and non-termination. Executing Mobile programs requires no change to existing .NET virtual machine implementations, since Mobile programs consist of normal managed CIL code with extra typing annotations stored in .NET attributes.
\end{abstract}

\section{Introduction}

Language-based approaches to computer security have employed two major strategies for enforcing security policies over untrusted programs.

- Low-level type systems, such as those used in Java bytecode [17], .NET CIL [7], and TAL for x86 [19], can enforce important program invariants such as memory safety and control safety, which dictate that

${ }^{*}$ Supported in part by AFOSR grant F49620-03-1-0156, National Science Foundation Grants 0430161 and CCF-0424422 (TRUST), ONR Grant N00014-01-1-0968, and a grant from Intel Corporation. The views and conclusions contained herein are those of the authors and should not be interpreted as necessarily representing the official policies or endorsements, either expressed or implied, of these organizations or the U.S. Government. 
programs must access and transfer control only to certain suitable memory addresses throughout their executions. Proof-Carrying Code (PCC) 20] generalizes the type-safety approach by providing an explicit proof of safety in first-order logic.

- Execution Monitoring technologies such as Java and .NET stack inspection [12] [17, II.22.11], SASI [9], Polymer [1, and Naccio [10, use runtime checks to enforce temporal properties that can depend on the history of the program's execution. For example, SASI Java was used to enforce the policy that no program may access the network after it reads from a file [8]. For efficiency, execution monitors are often implemented as In-lined Reference Monitors (IRM's) [23], wherein the runtime checks are in-lined into the untrusted program itself to produce a self-monitoring program.

The IRM approach is capable of enforcing a large class of powerful security policies, including ones that that cannot be enforced with purely static type-checking [14]. In addition, IRM's can enforce a flexible range of policies, often allowing the code recipient to choose the security policy after the code is received, whereas static type systems and PCC usually enforce fixed security policies that are encoded into the type system or proof logic itself, and that therefore cannot be changed without changing the type system or certifying compiler.

But despite their power and flexibility, the rewriters that automatically embed IRM's into untrusted programs are typically trusted components of the system. Since rewriters tend to be large and complex when efficient rewriting is required or complex security policies are to be enforced, the rewriter becomes a significant addition to the system's trusted computing base.

In this paper, we present Mobile, an extension to the .NET CIL that makes it possible to automatically verify IRM's using a static type-checker. Mobile (MOnitorable BIL with Effects) is an extension of BIL (Baby Intermediate Language) [13], a substantial fragment of managed .NET CIL that was used to develop generics for .NET [16]. Mobile programs are CIL programs with additional typing annotations that track an abstract representation of program execution history. These typing annotations allow a type-checker to verify statically that the runtime checks in-lined into the untrusted program suffice to enforce a specified security policy. Once typechecked, the typing annotations can be erased, and the self-monitoring program can be safely executed as normal CIL code. This verification process allows a rewriter to be removed from the trusted computing base and replaced with a (simpler) type-checker. Even when the rewriter is small and therefore comparable in size to the type-checker, type-checking constitutes a useful level of redundancy that provides greater assurance than trusting 


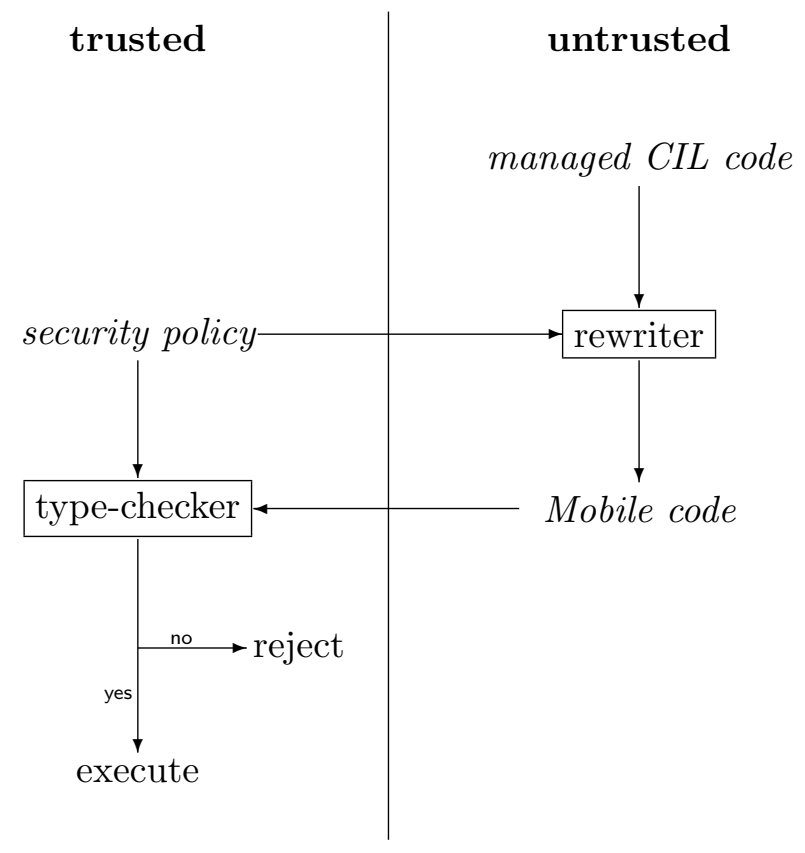

Figure 1: A Mobile load path

the rewriter alone. Mobile thus leverages the power of IRM's while using the type-safety approach to keep the trusted computing base small.

Figure 1 summarizes a typical load path on a system that executes IRM's written in Mobile. Untrusted, managed CIL code is first automatically rewritten according to a security policy, yielding a self-monitoring program written in Mobile. The rewriting can be performed by either a code producer or by a client machine receiving the untrusted code. Since the rewriter, and therefore the self-monitoring program, remains untrusted, the self-monitoring program is then passed to a trusted type-checker that certifies the code with respect to the original security policy. Code that satisfies the security policy will be approved by the type-checker, and is therefore safe to execute; code that is not well-typed will be rejected and would indicate a failure of the rewriter.

In this paper we focus on robust certification of Mobile code. Techniques for efficient rewriting are left to future work, but we describe a naïve rewriter and suggest some strategies for optimizing it in 83 . Our prototype implementation of Mobile consists of a type-checker that verifies sound rewriting with respect to security policies expressed as finite-state security automata. The implementation can verify both single-threaded and multithreaded managed CIL applications, and it supports language features beyond those modeled by BIL, such as exceptions and finalizers. 


\section{Related Work}

Type-systems $\lambda_{\mathcal{A}}$ [27] and $\lambda_{\text {hist }}$ [24] enforce history-based security policies over languages based on the $\lambda$-calculus. In both, program histories are tracked at the type-level using effect types that represent an abstraction of those global histories that might have been exhibited by the program prior to control reaching any given program point.

Mobile differs from $\lambda_{\mathcal{A}}$ and $\lambda_{\text {hist }}$ by tracking history on a per-object basis. That is, both $\lambda_{\mathcal{A}}$ and $\lambda_{\text {hist }}$ represent a program's history as a finite or infinite sequence of global program events, where the set of all possible global program events is always finite. Policies that are only expressible using an infinite set of global program events (e.g. events parameterized by object instances) are therefore not enforceable by $\lambda_{\mathcal{A}}$ or $\lambda_{\text {hist }}$. For example, the policy that every opened file must be closed by the time the program terminates is not enforceable by either $\lambda_{\mathcal{A}}$ or $\lambda_{\text {hist }}$ when the number of file objects that could be allocated during the program's execution is unbounded. In objectoriented languages such as the .NET CIL, policies concerning unbounded collections of objects arise naturally, so it is not clear how $\lambda_{\mathcal{A}}$ or $\lambda_{\text {hist }}$ can be extended to such settings.

Mobile, however, enforces policies that are universally quantified over objects of any given class. For example, a Mobile policy can dictate that, for each file handle object the program allocates, an Open operation must be performed on it before any Read operations can be performed on it. Mobile therefore allows objects to be treated as first-class in policy specifications, whereas $\lambda_{\mathcal{A}}$ and $\lambda_{\text {hist }}$ do not.

PCC has been proposed as a framework for supporting certifying rewriting using temporal logic [3]. The approach is potentially powerful, but does not presently support languages that include exceptions, concurrency, and other features found in real programming languages [2, p. 173]. It is therefore unclear whether proof size and verification speed would scale well in practical settings.

CQual [11 and Vault [6] are C-like languages that enforce history-based properties of objects by employing a flow-sensitive type system based on alias types [25]. Security-relevant objects in CQual or Vault programs have their base types augmented with type qualifiers, which track the securityrelevant state of the object. Control flow paths that include operations for changing the security state of an object at runtime cause the type qualifier of that object to change during type-checking. A type-checker can therefore determine if any object might enter a state at runtime that violates the security policy.

Vault's type system additionally includes variant types that allow a runtime value to reflect an object's current state. Untrusted programs can then test such values before performing security-relevant operations on the ob- 
jects they track. The Vault type-checker verifies that these runtime tests are sufficient to guard against a security violation by refining an object's type qualifier along control flow paths that test such a runtime value.

Inspired by CQual and Vault, our work scales these ideas up to a large existing programming language: the managed .NET CIL. In scaling up to a larger-scale language, we adopt a somewhat different approach to tracking object security states at the type level. Both CQual and Vault assign linear types to security-relevant objects (and, in the case of Vault, to runtime state values), and use aliasing analyses to track changes to items with linear types. However, it is not clear how such analyses can be extended to support concurrency or to support an important technique commonly used by IRM's to track object security states, wherein security-relevant objects are paired with runtime values that record their states, and then such pairs are permitted to leak to the heap. Existing alias analyses cannot easily track items that are permitted to leak to the heap arbitrarily, or that can be manipulated by multiple concurrent threads of execution.

We therefore take the approach of $\mathrm{L}^{3}$ [18], wherein linearly-typed items are permitted to leak to the heap by packing them into shared data structures with limited interfaces. These shared object-state pairs, called packages, can be aliased arbitrarily and are not tracked by the type system. Mobile provides trusted operations for packing and unpacking linear-typed items to and from shared package objects. To perform any (security-relevant) operation that might change a value with linear type, it must first be unpacked from any package that contains it. As with ownership types [5, 4, packing and unpacking operations are implemented as destructive reads, so that only one thread can perform security-relevant operations on a given security-relevant object at a time. By including appropriate pairing and unpairing operations in the code, IRM's can exploit the power of unrestricted aliasing, yet prove through the type system that all security-relevant objects are sufficiently monitored. Mobile's type system and the CLI permissions system are both leveraged to maintain invariants linking an object to an accurate runtime representation of its state.

\section{Overview}

A Mobile security policy identifies a set of security-relevant object classes and assigns a set of acceptable traces to each such class. A trace is a finite or infinite sequence of security-relevant events - program operations that take a security-relevant object as an argument. A Mobile program satisfies the security policy if (i) for every finite control flow path, the sequence of security-relevant events performed on every object allocated along that path is a member of the set of traces that the security policy has assigned to that object's class; and (ii) for every infinite control flow path, the sequence 
of security-relevant operations performed on every security-relevant object allocated along that path is a prefix of a member of the set of traces assigned to that object's class.

For example, a security policy that concerns files might identify the System.IO.File class provided by the .NET Common Language Runtime (CLR) as a security-relevant class, and might identify calls to the Open, Read, and Close methods of that class ${ }^{1}$ as security-relevant operations. A security policy that requires programs to open files before reading them, allows at most three reads per opened file, and requires programs to close files before the program terminates, might assign $\left(O\left(R \cup R^{2} \cup R^{3}\right) C\right)^{\omega}$ as the set of acceptable traces for class System. IO.File (where O, R, and C denote Open, Read, and Close events, respectively, and $\omega$ denotes finite or infinite repetition).

Although Mobile security policies model events as operations performed on objects, global events that do not concern any particular object can be encoded as operations on a global object that is allocated at program start and destroyed at program termination. Thus, Mobile policies can regard global events, per-object events, and combinations of the two.

For example, one might modify the example policy above by additionally requiring that at most ten network sends may occur during the lifetime of the program. In that case, the global object would additionally be identified as a security-relevant object, a Send method call performed on any System. Net. Sockets. Socket object would be identified as a securityrelevant event for the global object, and the global object would be assigned the set of traces denoted by $\epsilon \cup S \cup S^{2} \cup \cdots \cup S^{10}$ (where $S$ denotes a Send event).

A rewriter that produces self-monitoring programs from untrusted CIL code is expected to produce well-typed Mobile code, so that the policyadherence theorem can be used to guarantee that it is safe to execute. For this rewriting task to be feasible, Mobile's type system must be flexible enough to permit rewriters to insert runtime security checks - well-typed code that tracks the state of security-relevant objects at runtime, testing aspects of the state that cannot be verified statically. To that end, Mobile supports a pack operation that pairs a security-relevant object with a runtime value (e.g. an integer) representing an abstraction of the object's current state, and that encapsulates them into a two-field package object. Mobile's unpack operation can be used to unpack a package, yielding the original object that was packed along with the runtime value that represents its state. Mobile programs can then test this runtime value to infer information about the associated object's state. Both pack and unpack are implemented as

\footnotetext{
${ }^{1}$ The .NET CLR's File class does not actually have methods with these names, but instead supports file I/O via other classes such as the StreamReader class. We use more typical names to clarify the example.
} 
CIL method calls to a small trusted library (about ten lines of C\# code).

To keep type-checking tractable, Mobile does not allow security-relevant operations on objects that are packed. A package class' two fields are declared to be private so that, to access a security-relevant object directly and perform operations on it, it must first be unpacked. While unpacked, Mobile allows only limited aliasing of security-relevant objects - none of their aliases can escape to the heap. To enforce this restriction, the unpack operation is implemented as a destructive read, preventing the package from being unpacked again before it is re-packed. Packages, however, are permitted to escape to the heap and to undergo unlimited aliasing. These restrictions allow the type-checker to statically track histories of unpacked objects and to ensure that packed objects are always paired with a value that accurately reflects their state. When an object is packed, it is safe for the type-checker to forget whatever information might be statically known about the object, keeping the type-checking algorithm tractable and affording the rewriter a dynamic fallback mechanism when static analysis cannot verify all securityrelevant operations.

When pack and unpack are implemented as atomic operations, Mobile can also enforce security policies in concurrent settings. In such a setting, Mobile's type system maintains the invariant that each security-relevant object is either packed or held by at most one thread. Packed objects are always policy-adherent (or their finalizers must bring them to a policy-adherent state at program termination; see \$5), whereas unpacked objects are tracked by the type system to ensure that they return to a policy-adherent state before they are relinquished by the thread.

Using the above operations, a naïve rewriter can implement state-based histories by simply representing security-relevant objects as packages. Whenever a security-relevant operation is to be performed, the rewriter would insert code to first unpack the package and test the object's runtime state, then perform the security-relevant operation only if the test succeeds (possibly terminating otherwise), and finally repackage the object with updated state.

This strategy suffices to implement any state-based history but might result in inefficient code if security-relevant operations are frequent. Thus, Mobile's type system also makes it possible to avoid some of these dynamic operations when policy-adherence can be proved statically. For example, a more sophisticated rewriter could in some cases insert code to perform numerous security-relevant operations consecutively without any dynamic checks. Instead of dynamic checks, the rewriter could add typing annotations that prove to the type-checker that the omitted checks are unnecessary for preventing a security violation. Substituting annotations for dynamic checks in this way is often possible in straight-line code or tight loops that do not leak security-relevant objects to the heap. However, when objects 
do escape to the heap, the type system is not sufficiently powerful to track them and dynamic checks would usually be necessary in order to prove that a security violation cannot occur. Thus, Mobile's type system is sufficiently expressive that rewriters can avoid some but not all dynamic checks.

Our implementation of Mobile models security policies as finite-state security automata. This approach is appealing because it is simple, practical, it introduces minimal extra state to untrusted programs, and it seems to cover most of the enforceable security policies discussed in the literature. However, the formalisms presented in this paper do not assume any particular method of representing object states at runtime. Rather, we parameterize the framework in terms of arbitrary state representations and state tests so that alternative implementations can be realized in the future. For example, future implementations might track object states using LTL expressions or even by recording an object's complete history at runtime. Thus, Mobile constitutes a framework general enough to reason about many different in-lining strategies used by IRM's.

\section{A Formal Analysis of Mobile}

\subsection{The Abstract Machine}

Figure 2 gives the Mobile instruction set. Like BIL, Mobile's syntax is written in postfix notation. In addition to BIL instructions 2 , Mobile includes

- instruction evt $e$, which performs security-relevant operation $e$ on an object,

- instructions newpackage and newhist for creating packages and runtime state values,

- instructions pack and unpack for packing/unpacking objects and runtime state values to/from packages,

- instruction condst, which dynamically tests a runtime state value, and

- the pseudo-instructions $v$ and ret, which do not appear in source code but are introduced in the intermediate stages of the small-step semantics presented in 4.2 . (Instruction $v$ is a term that has been reduced to value $v$, and instruction ret pops the current stack frame at the end of a method call.)

\footnotetext{
${ }^{2}$ For simplicity, we omit BIL's value classes and managed pointers from Mobile, but otherwise include all BIL types and instructions.
} 


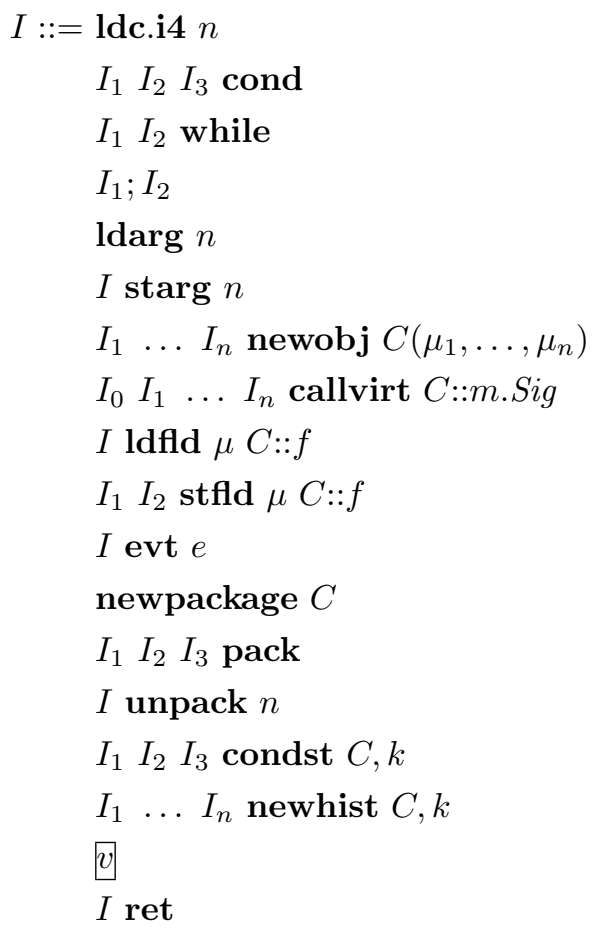

integer constant conditional while-loop sequence method argument store into arg make new obj method call load from field store into field exhibit event make new package pack package unpack package test state state constructor values* method return*

*Values and return instructions do not appear in Mobile source code, but are introduced by the small-step operational semantics as the program evaluates.

Figure 2: The Mobile instruction set

Types

Untracked types

Class names

Object identity variables

History abstractions

History abstraction variables

Method signatures

Typing contexts

Object history maps

Local variable frames

$$
\begin{aligned}
& \tau::=\mu \mid C\langle\ell\rangle \\
& \mu::=\text { void }|\operatorname{int32}| C\langle ?\rangle \mid \operatorname{Rep}_{C}\langle H\rangle \\
& C \\
& \ell \begin{array}{c}
H:= \\
\quad \epsilon|e| H_{1} H_{2}\left|H_{1} \cup H_{2}\right| H^{\omega} \mid \\
\quad \theta \mid H_{1} \cap H_{2}
\end{array}
\end{aligned}
$$

$\theta$

$$
\begin{gathered}
\text { Sig }::=\forall \Gamma_{\text {in }} \cdot\left(\left(\Psi_{\text {in }}, F r_{\text {in }}\right) \multimap\right. \\
\left.\exists \Gamma_{\text {out }} \cdot\left(\Psi_{\text {out }}, F r_{\text {out }}, \tau\right)\right) \\
\Gamma::=\cdot|\Gamma, \ell: C| \Gamma, \ell: C\langle ?\rangle \mid \Gamma, \theta \\
\Psi::=1 \mid \Psi \star(\ell \mapsto H) \\
\operatorname{Fr}::=\left(\tau_{0}, \ldots, \tau_{n}\right)
\end{gathered}
$$

Figure 3: The Mobile type system 


$$
\begin{gathered}
\overline{\tau \preceq \tau} \\
H \subseteq H^{\prime} \\
\frac{\operatorname{Rep}_{C}\langle H\rangle \preceq \operatorname{Rep}_{C}\left\langle H^{\prime}\right\rangle}{\tau_{i} \preceq \tau_{i}^{\prime} \forall i \in 0 . . n} \\
\frac{\operatorname{Dom}(\Psi)=\operatorname{Dom}\left(\Psi^{\prime}\right) \quad \Psi(\ell) \subseteq \tau^{\prime}(\ell) \forall \ell \in \operatorname{Dom}(\Psi)}{\left(\tau_{0}, \ldots, \tau_{0}^{\prime}, \ldots, \tau_{n}^{\prime}\right)} \\
\Psi \preceq \Psi^{\prime}
\end{gathered}
$$

Figure 4: Mobile subtyping

Figure 3 provides Mobile's type system. Mobile types consist of void types, integers, classes, and history abstractions (the types of runtime state values). The type of each unpacked, security-relevant object $C\langle\ell\rangle$ is parameterized by an object identity variable $\ell$ that uniquely identifies the object. All aliases of the object have types with the same object identity variable, but other unpacked objects of the same class have types with different object identity variables. The types $C\langle ?\rangle$ of packed classes and security-irrelevant classes do not include object identity variables, and their instances are therefore not distinguishable by the type system. We consider Mobile terms to be equivalent up to alpha conversion of bound variables.

The types $\operatorname{Rep}_{C}\langle H\rangle$ of runtime state values are parameterized both by the class type $C$ of the object to which they refer and by a history abstraction $H$-an $\omega$-regular expression (plus variables and intersection) that denotes a set of traces. In such an expression, $\omega$ denotes finite or infinite repetition.

Closed (i.e. variable-less) history abstractions conform to a subset relation; we write $H_{1} \subseteq H_{2}$ if the set of traces denoted by $H_{1}$ is a subset of the set of traces denoted by $\mathrm{H}_{2}$. This subset relation induces a natural subtyping relation $\preceq$ given in Figure 4 . Observe that the subtyping relation in Figure 4 does not recognize class subtyping of security-relevant classes. We leave support for subtyping of security-relevant classes to future work.

Type variables in Mobile types are bound by typing contexts $\Gamma$, which assign class or package types to object identity variables $\ell$ and declare any history abstraction variables $\theta$. Object identity variables can additionally appear in object history maps $\Psi$, which associate a history abstraction $H$ with each object identity variable that corresponds to an unpacked, securityrelevant object. Since object identity variables uniquely identify each object instance, object history maps can be seen as a spatial conjunction $(\star)$ [21] of assertions about the histories of the various unpacked objects in the heap. 


\begin{tabular}{|c|c|}
\hline$v::=$ & result \\
\hline 0 & void \\
\hline$i_{4}$ & integer \\
\hline$\ell$ & heap pointer \\
\hline $\operatorname{rep}_{C}(H)$ & runtime state value \\
\hline$o::=$ & heap elements \\
\hline$o b j_{C}\left\{f_{i}=v_{i}\right\}^{\vec{e}}$ & object \\
\hline$p k g\left(\ell, \operatorname{rep}_{C}(H)\right)$ & filled package \\
\hline$p k g(\cdot)$ & empty package \\
\hline$h::=\ell_{i} \mapsto o_{i}$ & heap \\
\hline$a::=\left(v_{0}, \ldots, v_{n}\right)$ & arguments \\
\hline$s::=\left(a_{0}, \ldots, a_{n}\right)$ & stack \\
\hline$\psi::=(h, s)$ & small-step store \\
\hline
\end{tabular}

Figure 5: The Mobile memory model

A complete Mobile program consists of:

$\begin{array}{ll}\text { Class names } & C \\ \text { Field types } & \text { field }:(C \times f) \rightarrow \mu \\ \text { Class methods } & \text { methodbody }:(\text { C::m.Sig }) \rightarrow I \\ \text { Class policies } & \text { policy }: C \rightarrow H\end{array}$

We also use the notation fields $(C)$ to refer to the number of fields in class C. Method signatures Sig will be described in $\$ 4.3$.

\subsection{Operational Semantics}

Unlike [13, we provide a small-step operational semantics for Mobile rather than a large-step semantics, so as to apply the policy adherence theorems presented in 4.4 to programs that do not terminate or that enter a bad state.

In Mobile's small-step memory model, presented in Figure 5, objects consist not only of an assignment of values to fields but also a trace $\vec{e}$ that records a history of the security-relevant operations performed on the object. Although our model attaches a history trace to each object, we prove in 4.4 that it is unnecessary for the virtual machine to track and store object traces because well-typed Mobile code never exhibits a trace that violates the security policy.

The small-step operational semantics of Mobile, given in Figures 6 and 7 , define how a given store $\psi$ and instruction $I$ steps to a new store $\psi^{\prime}$ and 


$$
\begin{aligned}
E::= & {[] \mid E I_{2} I_{3} \text { cond }\left|E ; I_{2}\right| E \operatorname{starg} n \mid } \\
& v_{1} \ldots v_{m} E I_{1} \ldots I_{n} \text { newobj } C\left(\mu_{1}, \ldots, \mu_{m+n+1}\right) \mid \\
& v_{1} \ldots v_{m} E I_{1} \ldots I_{n} \text { callvirt } C:: m . S i g \mid E \text { ret } \mid \\
& E \text { ldfld } \mu C:: f \mid E I_{2} \text { stfld } \mu C:: f \mid v_{1} E \text { stfld } \mu C:: f \mid \\
& E \text { evt } e \mid E I_{2} I_{3} \text { pack } \mid v_{1} E I_{3} \text { pack } \mid v_{1} v_{2} E \text { pack } \mid \\
& E \text { unpack } C, k \mid E I_{2} I_{3} \text { condst } C, k \mid \\
& v_{1} \ldots v_{m} E I_{1} \ldots I_{n} \text { newhist } C, k
\end{aligned}
$$

Figure 6: Mobile Evaluation Contexts

instruction $I^{\prime}$, written $\psi, I \rightsquigarrow \psi^{\prime}, I^{\prime}$.

Rules 17 and 18 use notation not previously defined and therefore deserve special note. Runtime operations test $C, k$ and hc $C, k$ test runtime state values and construct new runtime state values, respectively. Rather than fixing these two operations, we allow Mobile to be extended with unspecified implementations of them. Different implementations of test $C, k$ and hc ${ }_{C, k}$ can therefore be used to allow Mobile to support different collections of security policies. For example, a Mobile system that supports security policies expressed as DFA's might implement runtime state values as 32-bit integers and might support tests that compare runtime state values to integer constants (to determine which state the DFA is in). In that case, one could define for each $k \in 0 . .2^{32}$, hc $_{C, k}()=k$ and $\operatorname{test}_{C, k}(i)=\{1$ if $i=k$, else 0$\}$. A more powerful (but more computationally expensive) Mobile system might implement runtime state values as dynamic data structures that record an object's entire trace and might provide tests to examine such structures. In this paper, we assume only that a countable collection of state value constructors and tests exists and that this collection adheres to typing constraints 19, 20, 21, and 22 presented in 4.3 .

The operational semantics given in Figure 7 are for a single-threaded virtual machine without support for finalizers. To model concurrency, one could extend our stacks to consist of multiple threads and add a smallstep rule that non-deterministically chooses which thread to execute next. Finalizers could be modeled by adding another small-step rule that nondeterministically forks a finalizer thread whenever an object is unreachable. Our implementation supports concurrency and finalizers, but to simplify the presentation, we leave the analysis of these language features to future work.

\subsection{Type System}

Mobile's type system considers each Mobile term to be a linear operator from a history map and frame list (describing the initial heap and stack, 


$$
\begin{aligned}
& \psi, \text { ldc.i4 } i 4 \rightsquigarrow \psi, i_{4} \\
& \frac{\psi, I \rightsquigarrow \psi^{\prime}, I^{\prime}}{\psi, E[I] \rightsquigarrow \psi^{\prime}, E\left[I^{\prime}\right]} \\
& \text { if } i_{4}=0 \text { then } j=3 \text { else } j=2 \\
& \psi, i_{4} I_{2} I_{3} \text { cond } \rightsquigarrow \psi, I_{j} \\
& \psi, I_{1} I_{2} \text { while } \rightsquigarrow \psi, I_{1}\left(I_{2} ;\left(I_{1} I_{2} \text { while }\right)\right) 0 \text { cond } \\
& \psi, v ; I_{2} \rightsquigarrow \psi, I_{2} \\
& 0 \leq j \leq n \\
& \left(h, s\left(v_{0}, \ldots, v_{n}\right)\right), \operatorname{ldarg} j \rightsquigarrow\left(h, s\left(v_{0}, \ldots, v_{n}\right)\right), v_{j} \\
& 0 \leq j \leq n \\
& \left(h, s\left(v_{0}, \ldots, v_{n}\right)\right), v \operatorname{starg} j \rightsquigarrow \\
& \left(h, s\left(v_{0}, \ldots, v_{j-1}, v, v_{j+1}, \ldots, v_{n}\right)\right), \mathbf{0}
\end{aligned}
$$

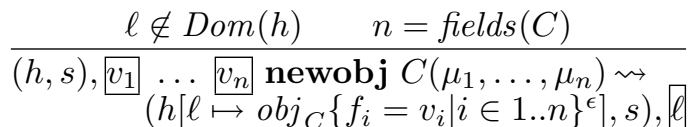

$$
\begin{aligned}
& \text { methodbody }(C:: \text { m.Sig })=I \\
& \overline{(h, s), v_{0} \ldots \sqrt{v_{n}} \text { callvirt } C:: m . S i g \rightsquigarrow\left(h, s\left(v_{0}, \ldots, v_{n}\right)\right), I \text { ret }} \\
& (h, s a), v \text { ret } \rightsquigarrow(h, s), v \\
& h(\ell)=o b j_{C}\{\ldots, f=v, \ldots\}^{\vec{e}} \\
& (h, s), \ell \text { ldfld } \mu C:: f \rightsquigarrow(h, s), v \\
& \frac{h(\ell)=o b j_{C}\{\ldots, f=v, \ldots\}^{\vec{e}}}{(h, s), \ell \ell\left[v^{\prime} \text { stfld } \mu C:: f \rightsquigarrow\left(h\left[\ell \mapsto o b j_{C}\left[f \mapsto v^{\prime}\right]\right], s\right), \boldsymbol{0}\right.} \\
& h(\ell)=o b j_{C}\{\ldots\}^{\vec{e}} \\
& \overline{(h, s), \ell \operatorname{evt} e_{1} \rightsquigarrow\left(h\left[\ell \mapsto o b j_{C}\{\ldots\}^{\vec{e} e_{1}}\right], s\right), \mathbf{0}} \\
& \ell \notin \operatorname{Dom}(h) \\
& (h, s) \text {, newpackage } C \rightsquigarrow(h[\ell \mapsto p k g(\cdot)], s), \ell \\
& \frac{h(\ell)=p k g(\ldots)}{(h, s), \ell \ell \ell^{\prime} \operatorname{rep} p_{C}(H) \text { pack } \rightsquigarrow\left(h\left[\ell \mapsto p k g\left(\ell^{\prime}, r e p_{C}(H)\right)\right], s\right), 0} \\
& h(\ell)=p k g\left(\ell^{\prime}, \operatorname{rep}_{C}(H)\right) \quad 0 \leq j \leq n \\
& \left(h, s\left(v_{0}, \ldots, v_{n}\right)\right), \ell \ell \text { unpack } j \rightsquigarrow \\
& \left(h[\ell \mapsto p k g(\cdot)], s\left(v_{0}, \ldots, v_{j-1}, \operatorname{rep}_{C}(H), v_{j+1}, \ldots, v_{n}\right)\right), \ell^{\prime} \\
& \text { if test }{ }_{C, k}\left(\operatorname{rep}_{C}(H)\right)=0 \text { then } j=3 \text { else } j=2 \\
& \psi, \operatorname{rep}_{C}(H) I_{2} I_{3} \text { condst } C, k \rightsquigarrow \psi, I_{j} \\
& \operatorname{arity}\left(\mathrm{hc}_{C, k}\right)=n \\
& \psi, v_{1} \ldots v_{n} \text { newhist } C, k \rightsquigarrow \psi, \mathrm{hc}_{C, k}\left(v_{1}, \ldots, v_{n}\right)
\end{aligned}
$$

Figure 7: Small-step Operational Sematics for Mobile 
1 (newobj $C()$ ) starg 1 ;

2 (ldarg 1) evt $e_{1}$;

3 (ldarg 1) evt $e_{2}$;

4 (newpackage $C$ ) stfld 2;

5 (ldarg 2) (ldarg 1) (newhist $C, 0$ ) pack;

6 (...) (ldarg 2) stfld ...;

7 ((ldarg 2) unpack 4) starg 3;

8 (ldarg 3$)\left(\left(\right.\right.$ ldarg 4) evt $\left.e_{1}\right)(\ldots)$ condst $C, 0$

Figure 8: Sample Mobile program

respectively) to a new history map and frame list (describing the heap and stack yielded by the operation) along with a return type. That is, we write $\Gamma \vdash I:(\Psi ; \overrightarrow{F r}) \multimap \exists \Gamma^{\prime} .\left(\Psi^{\prime} ; \overrightarrow{F r}^{\prime} ; \tau^{\prime}\right)$ if term $I$, when evaluated in typing context $\Gamma$, takes history map $\Psi$ and frame list $\overrightarrow{F r}$ (in which any typing variables are bound in context $\Gamma$ ) to new history map $\Psi^{\prime}$ and new frame list $\overrightarrow{F r}^{\prime}$, and yields a value of type $\tau^{\prime}$ (if it terminates). Any new typing variables appearing in $\overrightarrow{F r}^{\prime}$ and $\tau^{\prime}$ are bound in context $\Gamma^{\prime}$. A method signature (see Figure 3) is the type assigned to the term comprising its body.

Below, we provide an informal description of Mobile's typing rules by walking the type-checking algorithm through the sample Mobile program given in Figure 8. A complete list of typing rules is stated formally in the appendix.

Line 1 of the sample program creates a new object of class $C$ and stores it in local register 1 . When a new security-relevant object is created, Mobile's type system assigns it a fresh object identity variable $\ell$. The return type of the the newly created object is thus $C\langle\ell\rangle$ and the new history map yielded by the operation satisfies $\Psi^{\prime}(\ell)=\epsilon$; that is, new objects are initially assigned the empty trace.

As security-relevant events are performed on the object, the type system tracks these changes by statically updating its history map to append these new events to the sequence it recorded in its history map. So for example, after processing lines $2-3$ of the sample program, which perform events $e_{1}$ and $e_{2}$ on the object in local register 1 , the type-checker's new history map would satisfy $\Psi^{\prime}(\ell)=e_{1} e_{2}$. At each point that a security-relevant event is performed, the type system ensures that the new trace satisfies a prefix of the security policy. For example, when type-checking line 3, the typechecker would verify that $e_{1} e_{2} \subseteq \operatorname{pre}(\operatorname{policy}(C))$, where policy $(C)$ denotes the set of acceptable traces assigned by the security policy to class $C$, and pre $(\operatorname{policy}(C))$ denotes the set of prefixes of members of set policy $(C)$.

Security-relevant objects of type $C\langle\ell\rangle$ are like typical objects except that they are not permitted to escape to the heap. That is, they cannot be assigned to object fields. In order to leak a security-relevant object to the heap, a Mobile program must first store it in a package using a pack in- 
struction. This requires three steps: (1) A package must be created via a newpackage instruction. (2) A runtime state value must be created that accurately reflects the state of the object to be packed. This is accomplished via the newhist instruction, which is described in more detail below. (3) Finally, the pack operation is used to store the object and the runtime state value into the package. Lines 4 and 5 of the sample program illustrate these three steps. Line 4 creates a new package and stores it in local register 2 . Line 5 then fills the package using the object in local register 1 along with a newly created runtime state value.

In order for Mobile's type system to accept a pack operation, it must be able to statically verify that the runtime state value is an accurate abstraction of the object being packed. That is, if the runtime state value has type $\operatorname{Rep}_{C}\langle H\rangle$, then the type system requires that $\Psi(\ell) \subseteq H$ where $\ell$ is the object identity variable of the object being packed. Additionally, since packed objects are untracked and therefore might continue to exist until the program terminates, packed objects must satisfy the security policy. That is, we require that $\Psi(\ell) \subseteq$ policy $(C)$.

Packages that contain security-relevant objects can leak to the heap, as illustrated by line 6 of the sample program, which stores the package to a field of some other object. Since only packed objects can leak to the heap, the restriction that packed objects must be in a policy-adherent state is a potential limitation of the type system. That is, it might often be desirable to leak an object that is not yet in a policy-adherent state to the heap, but later retrieve it and restore it to a policy-adherent state before the program terminates. In $\$ 5$ we show how Mobile implementations can use finalizer code to avoid this restriction and leak objects to the heap even when they are not yet in a policy-adherent state.

After a pack operation, the type system removes object identity variable $\ell$ from the history map. Hence, after line 5 of the sample program, $\Psi^{\prime}(\ell)$ is undefined and the object that was packed becomes inaccessible. If the program were to subsequently attempt to load from local register 1 (before replacing its contents with something else), the type-checker would reject the code because that register now contains a value with an invalid type. Object identity variable $\ell$ can therefore be thought of as a capability that has been revoked from the local scope and given to the package.

In order to perform more security-relevant events on an object, a Mobile program must first reacquire a capability for the object by unpacking the object from its package via an unpack instruction. Line 7 of the sample program unpacks the package in local register 2 , storing the extracted object in local register 3 and storing the runtime state value that was packaged with it in local register 4 . Since packages and the objects they contain are not tracked by the type system, the type system cannot statically determine the history of a freshly unpacked object. All that is statically known is that 
the runtime state value that will be yielded at runtime by the unpack instruction will be an accurate representation of the unpacked object's history. To reflect this information statically, the type system assigns a fresh object identity variable $\ell^{\prime}$ to the unpacked object and a fresh history variable $\theta$ to the unknown history. The unpacked object and runtime state value then have types $C\left\langle\ell^{\prime}\right\rangle$ and $\operatorname{Rep}_{C}\langle\theta\rangle$, respectively, and the new history map satisfies $\Psi^{\prime}\left(\ell^{\prime}\right)=\theta$. The type $C\langle$ ? $\rangle$ of a package can hence be thought of as an existential type binding type variables $\ell^{\prime}$ and $\theta$.

If the sample program were at this point to perform security-relevant event $e$ on the newly unpacked object, Mobile's type system would reject because it would be unable to statically verify that $\theta e \subseteq$ policy $(C)$ (since nothing is statically known about history $\theta$ ). However, a Mobile program can perform additional evt operations on the object by first dynamically testing the runtime state value yielded by the unpack operation. If a Mobile program dynamically tests a value of type $\operatorname{Rep}_{C}\langle\theta\rangle$, Mobile's type system can statically infer information about history $\theta$ within the branches of the conditional. For example, if a condst instruction is used to test a value with type $\operatorname{Re} p_{C}\langle\theta\rangle$ for equality with a value of type $\operatorname{Rep}_{C}\left\langle e_{1} e_{2}\right\rangle$, then in the positive branch of the conditional, the type system can statically infer that $\theta=e_{1} e_{2}$. If policy $(C)=\left(e_{1} e_{2}\right)^{\omega}$, then a Mobile program could execute $I$ evt $e_{1}$ within the positive branch of such a conditional (where $I$ is the object that was unpacked), because $e_{1} e_{2} e_{1} \subseteq \operatorname{pre}\left(\left(e_{1} e_{2}\right)^{\omega}\right)$; but the typechecker would reject a program that executed $I$ evt $e_{2}$ in the positive branch, since $e_{1} e_{2} e_{2} \not$ pre $\left(\left(e_{1} e_{2}\right)^{\omega}\right)$.

Mobile supports many possible schemes for representing histories at runtime and for testing them, so rather than fixing particular operations for constructing runtime state values and particular operations for testing them, we instead assume only that there exists a countable collection of constructors newhist $C, k$ and conditionals condst $C, k$ for all integers $k$, that construct runtime state values and test runtime state values (respectively) for objects of class $C$. We then abstractly define $H C_{C, k}(\ldots)$ to be the type $\operatorname{Rep}_{C}\langle H\rangle$ of a history value constructed using constructor $k$ for security-relevant class $C$, and we define $c t x_{C, k}^{+}(H, \Psi)$ and $c t x_{C, k}^{-}(H, \Psi)$ to be the object history maps that refine $\Psi$ in the positive and negative branches (respectively) of a conditional that performs test $k$ on a history value of type $\operatorname{Rep}_{C}\langle H\rangle$. Mobile supports any such refinement that is sound in the sense that

$$
\text { test }_{C, k}(H)=0 \Longrightarrow \Psi \preceq c t x_{C, k}^{-}(H, \Psi)(\ell)
$$

and

$$
\operatorname{test}_{C, k}(H) \neq 0 \Longrightarrow \Psi \preceq c t x_{C, k}^{+}(H, \Psi)(\ell)
$$

We further assume that each history type constructor $H C_{C, k}(\ldots)$ accurately reflects its runtime implementation, in the sense that for all history value 
types $\operatorname{Rep}_{C_{1}}\left\langle H_{1}\right\rangle, \ldots, \operatorname{Rep}_{C_{n}}\left\langle H_{n}\right\rangle$ such that $n=\operatorname{arity}\left(H C_{C, k}\right)$, there exists some $H$ such that

$$
H C_{C, k}\left(\operatorname{Rep}_{C_{1}}\left\langle H_{1}\right\rangle, \ldots, \operatorname{Rep}_{C_{n}}\left\langle H_{n}\right\rangle\right)=\operatorname{Rep}_{C}\langle H\rangle
$$

and

$$
\operatorname{hc}_{C, k}\left(\operatorname{rep}_{C_{1}}\left(H_{1}\right), \ldots, \operatorname{rep}_{C_{n}}\left(H_{n}\right)\right)=\operatorname{rep}_{C}(H)
$$

In the sample program, suppose that history value constructor newhist $C, 0$ takes no arguments and yields a runtime value that represents history $e_{1} e_{2}$; and suppose that conditional test condst $C, 0$ compares a runtime state value to the value that represents history $e_{1} e_{2}$. Formally, suppose that $H C_{C, 0}()=\operatorname{Rep}_{C}\left\langle e_{1} e_{2}\right\rangle$ and $c t x_{C, 0}^{+}(\theta, \Psi)=\Psi\left[\theta \mapsto e_{1} e_{2}\right]$. Thus, in the positive branch of such a test, the type-checker's object history map can be refined by substituting $e_{1} e_{2}$ for any instances of the history variable being tested. Then if policy $(C)=\left(e_{1} e_{2}\right)^{\omega}$, a Mobile type-checker would accept the sample program. In the positive branch of the conditional in line 8, the type-checker would infer that the object in local register 4 has history $e_{1} e_{2}$, and therefore it is safe to perform event $e_{1}$ on it. However, if $\operatorname{policy}(C)=e_{1} e_{2} e_{2}$, then the type-checker would reject, because $e_{1} e_{2} e_{1}$ is not a prefix of $e_{1} e_{2} e_{2}$.

Our implementation of Mobile implements history abstraction values as integers. Thus, it provides $2^{32}$ newhist operations for each security-relevant class $C$, defining $\mathrm{hc}_{C, k}()=k$ for all $k \in 0 . .2^{32}-1$. Tests condst of runtime state values are implemented as equality comparisons between the integer runtime state value to be tested and an integer constant. Thus, we define

$$
\begin{aligned}
\text { test }_{C, k}\left(\operatorname{rep}_{C}(\theta)\right) & = \begin{cases}1 & \text { if } \operatorname{rep}_{C}(\theta)=k \\
0 & \text { otherwise }\end{cases} \\
\operatorname{ctx}_{C, k}^{+}(\theta, \Psi) & =\Psi\left[\theta \mapsto \theta \cap H_{k}\right] \\
\operatorname{ctx}_{C, k}^{-}(\theta, \Psi) & =\Psi\left[\theta \mapsto \theta \cap\left(\cup_{i \neq k} H_{i}\right)\right]
\end{aligned}
$$

for each integer $k \in 0 . .2^{32}-1$, where $H_{k}$ is a closed history abstraction statically assigned to integer constant $k$. The assignments of closed history abstractions $H_{k}$ to integers $k$ are not trusted, so this mapping can be defined by the Mobile program itself (e.g., in settings where self-monitoring programs are produced by a common rewriter or where separately produced programs do not exchange objects) or by the policy-writer (in settings where the mapping must be defined at a system global level for consistency).

The above scheme allows a Mobile program to represent object security states at runtime with a security automaton of $2^{32}$ states or less. Each state of the automaton is assigned an integer constant $k$, and history abstraction 
$H_{k}$ would denote the set of traces that cause the automaton to arrive in state $k$.

Many other extensions to Mobile are also possible. For example, rather than implementing runtime state values as simple integers, they could be implemented as data structures that store LTL expressions or complete trace histories. Tests of these data structures could be implemented as calls to methods in a trusted library.

\subsection{Policy Adherence of Mobile Programs}

The operational semantics of Mobile presented in $\$ 4.2$ permit untyped Mobile programs to enter bad terminal states - states in which the Mobile program has not been reduced to a value but no progress can be made. For example, an untyped Mobile program might attempt to load from a non-existent field or attempt to unpack an empty package (in which case no small-step rule can be applied). Mobile's type system presented in \$4.3 prevents both policy violations and bad terminal states, except that it does not prevent unpack operations from being performed on empty packages. This reflects the reality that in practical settings there will always be bad terminal states that are not statically preventable. We prove below that Mobile programs well-typed with respect to a security policy will not violate the security policy when executed even if they enter a bad state.

Formally, we define well-typed by

Definition 1. A method C::m.Sig with $\operatorname{Sig}=\forall \Gamma_{i n} \cdot\left(\Psi_{i n}, F r_{i n}\right) \multimap$ $\exists \Gamma_{\text {out }} .\left(\Psi_{\text {out }}, F r_{\text {out }}, \tau\right)$ is well-typed if and only if there exists a derivation for the typing judgment $\Gamma_{\text {in }} \vdash I:\left(\Psi_{\text {in }}, F r_{\text {in }}\right) \multimap \exists \Gamma_{\text {out }} .\left(\Psi_{\text {out }}, F r_{\text {out }}, \tau\right)$ where $I=$ methodbody $(C:: m . S i g)$.

Definition 2. A Mobile program is well-typed if and only if (1) for all $C:: m . S i g \in \operatorname{Dom}($ methodbody), method C::m.Sig is well-typed, and (2) there exists a method $C_{\text {main }}::$ main.Sig ${ }_{\text {main }} \in$ Dom(methodbody) with Sig $_{\text {main }}=$ $\forall \Gamma_{\text {in }} .\left(\Psi_{\text {in }},\left(\tau_{1}, \ldots, \tau_{n}\right)\right) \multimap \exists \Gamma_{\text {out }} .\left(\Psi_{\text {out }}, F r_{\text {out }}, \tau_{\text {out }}\right)$ such that for all substitutions $\sigma: \theta \rightarrow \vec{e}$ and all object identity variables $\ell: C \in\left(\Gamma_{\text {in }}, \Gamma_{\text {out }}\right)$, if $\Psi_{\text {out }}(\ell)=H$ then $\sigma(H) \subseteq \operatorname{policy}(C)$.

Part 2 of definition 2 captures the requirement that a Mobile program's entry method must have a signature that complies with the security policy on exit.

Policy violations are defined differently depending on whether the program terminates normally. If the program terminates normally, Mobile's type system guarantees that the resulting heap will be policy-adherent; whereas if the program does not terminate or enters a bad state, Mobile guarantees only that the heap at each evaluation step will be prefix-adherent, where policy- and prefix-adherence are defined as follows: 
Definition 3 (Policy Adherent). A heap $h$ is policy-adherent if, for all class objects $\operatorname{obj}_{C}\{\ldots\}^{\vec{e}} \in R n g(h), \vec{e} \subseteq \operatorname{policy}(C)$.

Definition 4 (Prefix Adherent). A heap $h$ is prefix-adherent if, for all class objects $o b j_{C}\{\ldots\}^{\vec{e}} \in R n g(h), \vec{e} \subseteq \operatorname{pre}(\operatorname{policy}(C))$.

To formalize the theorem, we first define a notion of consistency between a static typing context and a runtime memory state. We say that a memory store $\psi$ respects an object identity context $\Psi$ and a list of frames $\overrightarrow{F r}$, written $\Gamma \vdash \psi:(\Psi ; \overrightarrow{F r})$ if there exists a derivation using the the inference rules given in Figure 9. The following two theorems then establish that well-typed Mobile programs do not violate the security policy.

Theorem 1 (Terminating Policy Adherence). Assume that a Mobile program is well-typed, and that, as per Definition 2, its main method has signature $\operatorname{Sig}_{\text {main }}=\forall \Gamma_{\text {in }} .\left(\Psi_{\text {in }},\left(\tau_{1}, \ldots, \tau_{n}\right)\right) \multimap \exists \Gamma_{\text {out }} .\left(\Psi_{\text {out }}, F r_{\text {out }}, \tau_{\text {out }}\right)$. If $\Gamma_{\text {in }} \vdash \psi:\left(\Psi_{\text {in }} ;\right.$ Fr $)$ holds and if $\psi$, methodbody $\left(C_{\text {main }}::\right.$ main.Sig $) \rightsquigarrow^{*}\left(h^{\prime}, s^{\prime}\right), v$ holds, then $h^{\prime}$ is policy-adherent.

Proof. See Appendix B.

Theorem 2 (Non-terminating Prefix Adherence). Assume that a Mobile program is well-typed, and assume that $\Gamma \vdash I:(\Psi ; \overrightarrow{F r}) \multimap$ $\exists \Gamma^{\prime} .\left(\Psi^{\prime} ; \overrightarrow{F r}^{\prime} ; \tau\right)$ and $\Gamma \vdash(h ; s):(\Psi ; \overrightarrow{F r})$ hold. If $h$ is prefix-adherent and $(h, s), I \rightsquigarrow^{n}\left(h^{\prime}, s^{\prime}\right), I^{\prime}$ holds, then $h^{\prime}$ is prefix-adherent.

Proof. See Appendix B.

An important consequence of both of these theorems is that Mobile can be implemented on existing .NET systems without modifying the memory model to store object traces at runtime. Since a static type-checker can verify that Mobile code is well-typed, and since well-typed code never exhibits a trace that violates the security policy, the runtime system need not store or monitor object traces to prevent security violations.

\section{Implementation}

Our prototype implementation of Mobile consists of a type-checker for Mobile's type system extended to the full managed subset of Microsoft's .NET CIL. The type-checker was written in Ocaml (about one thousand lines of code) and uses Microsoft's .NET ILX SDK [26] to read and manipulate .NET bytecode binaries. Mobile programs are .NET CIL programs with typing annotations encoded as .NET method attributes. The Mobile type-checker reads these (untrusted) annotations and verifies them in the course of type-checking. 


$$
\begin{aligned}
& \frac{\Gamma \vdash_{\text {heap }} h: \Gamma \quad \vdash_{\text {hist }} h:(\Gamma ; \Psi) \quad \Gamma \vdash_{\text {stack }} s: \overrightarrow{F r}}{\Gamma \vdash(h, s):(\Psi ; \overrightarrow{F r})} \\
& \Gamma_{0} \vdash_{\text {heap }} h: \Gamma \\
& \underline{\Gamma_{0} \vdash v_{i}:(\Psi ; \overrightarrow{F r}) \multimap\left(\Psi ; \overrightarrow{F r} ; \text { field }\left(C, f_{i}\right)\right) \forall i \in 1 . . \text { fields }(C)} \\
& \overline{\Gamma_{0} \vdash_{\text {heap }} h,\left(\ell \mapsto o b j_{C}\left\{f_{i}=v_{i} \mid i \in 1 . . \text { fields }(C)\right\}^{\vec{e}}\right): \Gamma, \ell: C} \\
& \frac{\Gamma_{0} \vdash_{\text {heap }} h: \Gamma}{\Gamma_{0} \vdash_{\text {heap }} h,(\ell \mapsto p k g(\ldots)): \Gamma, \ell: C\langle ?\rangle} \\
& \frac{\Gamma_{0} \vdash_{\text {heap }} h: \Gamma}{\Gamma_{0} \vdash_{\text {heap }} h: \Gamma, \theta} \\
& \overline{\Gamma_{0} \vdash_{\text {heap }} \cdot: \cdot} \\
& \frac{\vdash_{\text {hist }} h:(\Gamma ; \Psi) \quad \vec{e} \subseteq H}{\vdash_{\text {hist }} h,\left(\ell \mapsto o b j_{C}\{\ldots\}^{\vec{e}}\right):(\Gamma, \ell: C ; \Psi \star(\ell \mapsto H))} \\
& \begin{aligned}
\vdash_{\text {hist }} h:(\Gamma ; \Psi) \quad \vec{e} \subseteq H \subseteq \operatorname{policy}(C) \\
\vdash_{\text {hist }} h,\left(\ell \mapsto p k g\left(\ell^{\prime}, \operatorname{rep}_{C}(H)\right)\right),\left(\ell^{\prime} \mapsto \operatorname{obj}_{C}\{\ldots\}^{\vec{e}}\right): \\
\left(\Gamma, \ell: C\langle ?\rangle, \ell^{\prime}: C ; \Psi\right)
\end{aligned} \\
& \frac{\vdash_{\text {hist }} h:(\Gamma ; \Psi) \quad \vec{e} \subseteq \operatorname{policy}(C)}{\vdash_{\text {hist }} h,\left(\ell \mapsto o b j_{C}\{\ldots\}^{\vec{e}}\right):(\Gamma, \ell: C ; \Psi)} \\
& \frac{\vdash_{\text {hist }} h:(\Gamma ; \Psi)}{\vdash_{\text {hist }} h,(\ell \mapsto p k g(\cdot)):(\Gamma, \ell: C\langle ?\rangle ; \Psi)} \\
& \frac{\vdash_{\text {hist }} h:(\Gamma ; \Psi)}{\vdash_{\text {hist }} h:(\Gamma, \theta ; \Psi)} \\
& \overline{\vdash_{\text {hist }} \cdot:(\cdot ; 1)} \\
& \frac{\Gamma \vdash_{\text {stack }} s: \overrightarrow{F r} \quad \Gamma \vdash v_{i}:\left(\Psi ; \overrightarrow{F r}_{0}\right) \multimap\left(\Psi ; \overrightarrow{F r}_{0} ; \tau_{i}\right) \forall i \in 0 . . n}{\Gamma \vdash_{\text {stack }} s\left(v_{0}, \ldots, v_{n}\right): \overrightarrow{F r}\left(\tau_{0}, \ldots, \tau_{n}\right)} \\
& \overline{\Gamma \vdash_{\text {stack }} \cdot:}
\end{aligned}
$$

Figure 9: Consistency of Mobile Statics and Dynamics 


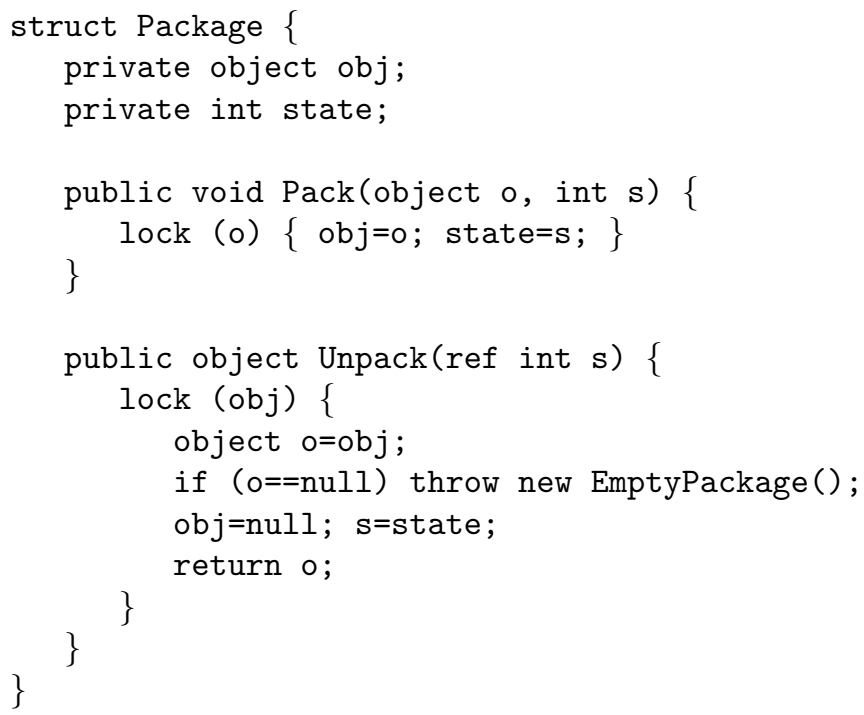

Figure 10: Implementation of pack and unpack

Our implementation allows security policies to identify method calls as security-relevant events. Thus, security policies can constrain the usage of resources provided by the CLR by monitoring CLR method calls and the objects they return. Our type-checker can, in principle, regard any CIL instruction as a security-relevant event, but we leave practical investigation of this feature to future work.

Operations pack and unpack are implemented as method calls to the (very small) trusted C\# library given in Figure 10. Observe that C\#'s lock construct is used to make both operations atomic. History abstraction values are implemented as integers. Thus, our newhist operation is simply a ldc.i4 instruction that loads an integer constant onto the evaluation stack. Policies can statically declare for each integer constant a closed history abstraction that integer represents when used as a runtime state value. Tests of runtime state values consist of equality comparisons with integer constants in the manner described in 4.3 .

With this simple support for history abstractions and tests, our typechecker can support IRM's that enforce security policies by expressing each object's state with a security automaton. Such an IRM can assign an integer constant to each state of the automaton, and can associate with each such constant a history abstraction that denotes the set of traces causing the automaton to enter the given state. The integer equality tests then allow the IRM to test whether any object's automaton is in any particular state.

The type-checker must verify subset relations over the language of history abstractions given in Figure 3. Although deciding subset for $\omega$-regular 
expressions with variables and intersection is not tractable in general, the task is simplified by observing that real Mobile code only introduces history variables at the beginnings of expressions (when an object is unpacked) and only introduces intersections that involve a variable and a closed history abstraction (when a runtime state value is tested). Furthermore, history variables cannot appear in policies or their prefixes, further reducing the possible forms. The resulting sub-language can be decided using a regular expression subset algorithm (see Appendix C).

Our type-checker also recognizes method annotations attached to finalizers of security-relevant classes. A finalizer's precondition must be satisfied whenever an object of its class escapes to the heap (i.e. when it is packed), since at any point after that, its package object could become orphaned and then garbage-collected. By the time a program terminates, all of its objects are guaranteed to satisfy their finalizers' postconditions, since at that point any remaining objects will be garbage-collected. This allows an IRM to leak security-relevant objects to the heap (in packages) even when they are not yet in a policy-adherent state, as long as the object's finalizer suffices to restore it to a policy-adherent state once garbage-collection occurs.

\section{Conclusions and Future Work}

Mobile's type system and the theorems presented in $\$ 4.4$ show that a common style of IRM, in which extra state variables and guards that model a security automaton have been in-lined into the untrusted code, can be independently verified by a type-checker, eliminating the need to trust the rewriter that produced the IRM. We verify policies that are universally quantified over unbounded collections of objects - that is, policies that require each object to exhibit a history of security-relevant events that conforms to some stated property. The language of security policies is left abstract and could consist of DFA's, LTL expressions, or any computable language of finite and infinite event sequences.

Our implementation of Mobile for managed Microsoft .NET CIL expresses security policies as finite-state security automata. We verify such policies in the presence of exceptions, concurrency, finalizers, and nontermination, demonstrating that Mobile can be scaled to real type-safe, low-level languages.

Our presentation of Mobile has not addressed issues of object inheritance of security-relevant classes. Future work should examine how to safely express and implement policies that require objects related by inheritance to conform to different properties. A type-checker for such a system would need to identify when a typecast at runtime could potentially lead to a violation of the policy and provide a means for policy-adherent programs to perform necessary typecasts. 
Another open problem is how to support a wider range of IRM implementations. Mobile supports only a specific (but typical) treatment of runtime state, wherein each security-relevant object is paired with a dynamic representation of its state every time it is leaked to the heap. In some settings, it may be desirable to implement IRM's that store an object's dynamic state differently, such as in a separate array rather than packaged together with the object it models. Type systems for coordinated data structures 22] could potentially be leveraged to support these decoupled objects and states, maintaining the invariant that security-relevant objects and the runtime state values that monitor them remain consistent with one another.

We chose a type system for Mobile that statically tracks control flow in a data-insensitive manner, with $\omega$-regular expressions denoting sets of event sequences. This approach is appealing because there is a natural rewriting strategy (outlined in $\$ 3$ ) whereby well-typed Mobile code can be automatically generated from untrusted CIL code. A more powerful type system could employ a richer language like Hoare Logic [15] to track data-sensitive control flow. This could allow clever rewriters to eliminate additional runtime checks by statically proving that they are unnecessary. However, formulating a sound and complete Hoare Logic for .NET that includes objects and concurrency is challenging; furthermore, the burden of producing useful proofs in this logic would be pushed to the rewriter. Future work should investigate rewriting strategies that could make such an approach worthwhile.

Finally, not every enforceable security policy can be couched as a computable property that is universally quantified over object instances. For example, one potentially useful policy is one that requires that for every file object opened for writing, there exists an encryptor object to which its output stream has been linked. Such a policy is not supported by Mobile because it regards both universal and existentially quantified properties that relate multiple object instances. Future work should consider how to implement IRM's that enforce such policies, and how these implementations could be type-checked so as to statically verify that the IRM satisfies the security policy.

\section{Acknowledgments}

The authors are indebted to Matthew Fluet, Michael Hicks, and Amal Ahmed for their helpful critiques of this paper.

\section{References}

[1] Lujo Bauer, Jay Ligatti, and David Walker. Composing security policies with polymer. In ACM SIGPLAN Conference on Programming 
Language Design and Implementation (PLDI), pages 305-314, Chicago, Illinois, June 2005.

[2] Andrew Bernard. Engineering Formal Security Policies for ProofCarrying Code. PhD thesis, Carnegie Mellon University, Pittsburgh, Pennsylvania, April 2004.

[3] Andrew Bernard and Peter Lee. Temporal logic for proof-carrying code. In 18th International Conference on Automated Deduction, pages 3146, Copenhagen, Denmark, July 2002.

[4] D. G. Clarke and S. Drossopoulou. Ownership, encapsulation and disjointness of type and effect. In 17th Annual ACM Conference on ObjectOriented Programming, Systems, Languages, and Applications (OOPSLA), pages 292-310, Seattle, Washington, November 2002.

[5] D. G. Clarke, J. Noble, and J. M. Potter. Simple ownership types for object containment. In European Conference for Object-Oriented Programming (ECOOP), pages 53-76, Budapest, Hungary, June 2001.

[6] Robert DeLine and Manuel Fähndrich. Enforcing high-level protocols in low-level software. In ACM SIGPLAN Conference on Programming Language Design and Implementation (PLDI), pages 59-69, Snowbird, Utah, June 2001.

[7] ECMA. ECMA-335: Common Language Infrastructure (CLI). ECMA (European Association for Standardizing Information and Communication Systems), Geneva, Switzerland, second edition, December 2002.

[8] Úlfar Erlingsson and Fred B. Schneider. IRM enforcement of Java stack inspection. In IEEE Symposium on Security and Privacy, pages 246255, Oakland, California, May 2000.

[9] Úlfar Erlingsson and Fred B. Schneider. SASI enforcement of security policies: A retrospective. In WNSP: New Security Paradigms Workshop. ACM Press, 2000.

[10] David Evans and Andrew Twynman. Flexible policy-directed code safety. In IEEE Symposium on Security and Privacy, pages 32-45, Oakland, California, May 1999.

[11] Jeffrey S. Foster, Tachio Terauchi, and Alex Aiken. Flow-sensitive type qualifiers. In ACM SIGPLAN Conference on Programming Language Design and Implementation (PLDI), pages 1-12, Berlin, Germany, June 2002.

[12] Li Gong. Java ${ }^{\mathrm{TM}} 2$ platform security architecture, version 1.2. Whitepaper. (c) 1997-2002 Sun Microsystems, Inc. 
[13] A. D. Gordon and D. Syme. Typing a multi-language intermediate code. In 28th ACM Symposium on Principles of Programming Languages, pages 248-260, London, United Kingdom, January 2001.

[14] Kevin W. Hamlen, Greg Morrisett, and Fred B. Schneider. Computability classes for enforcement mechanisms. To appear in ACM Transactions on Programming Languages and Systems, 2006. Also available as Technical Report TR-2003-1908, Cornell University, Ithaca, New York, August 2003.

[15] C.A.R. Hoare. An axiomatic basis for computer programming. Communications of the ACM, 12(10):576-580 and 583, October 1969.

[16] Andrew Kennedy and Don Syme. The design and implementation of generics for the .NET common language runtime. In ACM SIGPLAN Conference on Programming Language Design and Implementation (PLDI), pages 1-12, Snowbird, Utah, June 2001.

[17] Tim Lindholm and Frank Yellin. The Java ${ }^{\mathrm{TM}}$ Virtual Machine Specification. Addison-Wesley, second edition, 1999.

[18] Greg Morrisett, Amal Ahmed, and Matthew Fluet. L ${ }^{3}$ : A linear language with locations. To appear in the 7th International Conference on Typed Lambda Calculi and Applications.

[19] Greg Morrisett, Karl Crary, Neal Glew, Dan Grossman, Richard Samuels, Frederick Smith, David Walker, Stephanie Weirich, and Steve Zdancewic. TALx86: A realistic typed assembly language. In $A C M$ SIGPLAN Workshop on Compiler Support for System Software, pages 25-35, Atlanta, Georgia, May 1999.

[20] George C. Necula and Peter Lee. The design and implementation of a certifying compiler. In ACM SIGPLAN Conference on Programming Language Design and Implementation (PLDI), pages 333-344, 1998.

[21] Peter O'Hearn, John Reynolds, and Hongseok Yang. Local reasoning about programs that alter data structures. In 15th Annual Conference of the European Association for Computer Science Logic, LNCS, pages 1-19, Paris, France, 2001. Springer-Verlag.

[22] Michael F. Ringenburg and Dan Grossman. Types for describing coordinated data structures. In ACM SIGPLAN International Workshop on Types in Languages, Design and Implementation (TLDI), pages 25-36, Long Beach, California, January 2005.

[23] Fred B. Schneider. Enforceable security policies. ACM Transactions on Information and Systems Security, 3(1):30-50, February 2000. 
[24] Christian Skalka and Scott F. Smith. History effects and verification. In Asian Programming Languages Symposium (APLAS), pages 107-128, November 2004.

[25] Frederick Smith, David Walker, and Greg Morrisett. Alias types. Lecture Notes in Computer Science, 1782:366-381, March 20002000.

[26] Don Syme. ILX: Extending the .NET Common IL for functional language interoperability. In Nick Benton and Andrew Kennedy, editors, First International Workshop on Multi-Language Infrastructure and Interoperability, volume 59.1, Florence, Italy, September 2001.

[27] David Walker. A type system for expressive security policies. In 27th ACM SIGPLAN Symposium on Principles of Programming Languages, pages 254-267, January 2000.

\section{A Typing Rules}

The following is a formal statement of Mobile's typing rules.

$$
\begin{aligned}
& \overline{\Gamma \vdash \text { ldc.i4 } n:(\Psi ; \overrightarrow{F r}) \multimap(\Psi ; \overrightarrow{F r} ; \text { int32 })} \\
& \Gamma \vdash I_{1}:(\Psi ; \overrightarrow{F r}) \multimap \exists \Gamma_{1} \cdot\left(\Psi_{1} ; \overrightarrow{F r}_{1} ; \text { int32 }\right) \\
& \underline{\Gamma, \Gamma_{1} \vdash I_{i}:\left(\Psi_{1} ; \overrightarrow{F r}_{1}\right) \multimap \exists \Gamma^{\prime} .\left(\Psi^{\prime} ; \overrightarrow{F r}^{\prime} ; \tau\right) \forall i \in\{2,3\}} \\
& \Gamma \vdash I_{1} I_{2} I_{3} \text { cond }:(\Psi ; \overrightarrow{F r}) \multimap \exists \Gamma_{1}, \Gamma^{\prime} .\left(\Psi^{\prime} ; \overrightarrow{F r}^{\prime} ; \tau\right) \\
& \frac{\Gamma, \Gamma^{\prime} \vdash I_{1} I_{2} \text { 0 cond }:(\Psi ; \overrightarrow{F r}) \multimap \exists \Gamma^{\prime} .(\Psi ; \overrightarrow{F r} ; \text { void })}{\Gamma, \Gamma^{\prime} \vdash I_{1} I_{2} \text { while }:(\Psi ; \overrightarrow{F r}) \multimap \exists \Gamma^{\prime} .(\Psi ; \overrightarrow{F r} ; \text { void })} \\
& \Gamma \vdash I_{1}:(\Psi ; \overrightarrow{F r}) \multimap \exists \Gamma_{1} \cdot\left(\Psi_{1} ; \overrightarrow{F r}_{1} ; \text { void }\right) \\
& \Gamma, \Gamma_{1} \vdash I_{2}:\left(\Psi_{1} ; \overrightarrow{F r}_{1}\right) \multimap \exists \Gamma_{2} \cdot\left(\Psi^{\prime} ; \overrightarrow{F r}^{\prime} ; \tau\right) \\
& \Gamma \vdash I_{1} ; I_{2}:(\Psi ; \overrightarrow{F r}) \multimap \exists \Gamma_{1}, \Gamma_{2} \cdot\left(\Psi^{\prime} ; \overrightarrow{F r}^{\prime} ; \tau\right) \\
& \ell \in \operatorname{Dom}\left(\Psi^{\prime}\right) \quad \text { field }(C, f)=\mu \\
& \Gamma \vdash I:(\Psi ; \overrightarrow{F r}) \multimap \exists \Gamma^{\prime} .\left(\Psi^{\prime} ; \overrightarrow{F r}^{\prime} ; C\langle\ell\rangle\right) \\
& \overrightarrow{\Gamma \vdash I \text { ldfld } \mu C:: f:(\Psi ; \overrightarrow{F r}) \multimap \exists \Gamma^{\prime} .\left(\Psi^{\prime} ; \overrightarrow{F r}^{\prime} ; \mu\right)} \\
& \Gamma \vdash I_{1}:(\Psi ; \overrightarrow{F r}) \multimap \exists \Gamma_{1} .\left(\Psi_{1} ; \overrightarrow{F r}_{1} ; C\langle\ell\rangle\right) \quad \ell \in \operatorname{Dom}\left(\Psi^{\prime}\right) \\
& \underline{\Gamma, \Gamma_{1} \vdash I_{2}:\left(\Psi_{1} ; \overrightarrow{F r}_{1}\right) \multimap \exists \Gamma_{2} .\left(\Psi^{\prime} ; \overrightarrow{F r}^{\prime} ; \mu\right) \quad \text { field }(C, f)=\mu} \\
& \Gamma \vdash I_{1} I_{2} \text { stfld } \mu C:: f:(\Psi ; \overrightarrow{F r}) \multimap \exists \Gamma_{1}, \Gamma_{2} .\left(\Psi^{\prime} ; \overrightarrow{F r}^{\prime} ; \text { void }\right) \\
& \frac{0 \leq j \leq n}{\Gamma \vdash \operatorname{ldarg} j:\left(\Psi ; \overrightarrow{F r}\left(\tau_{0}, \ldots, \tau_{n}\right)\right) \multimap\left(\Psi ; \overrightarrow{F r}\left(\tau_{0}, \ldots, \tau_{n}\right) ; \tau_{j}\right)} \\
& \frac{\Gamma \vdash I:(\Psi ; \overrightarrow{F r}) \multimap \exists \Gamma^{\prime} .\left(\Psi^{\prime} ; \overrightarrow{F r}^{\prime}\left(\tau_{0}, \ldots, \tau_{n}\right) ; \tau\right) \quad 0 \leq j \leq n}{\Gamma \vdash I \operatorname{starg} j:(\Psi \cdot \overrightarrow{F r}) \multimap} \\
& \exists \Gamma^{\prime} .\left(\Psi^{\prime} ; \overrightarrow{F r}^{\prime}\left(\tau_{0}, \ldots, \tau_{j-1}, \tau, \tau_{j+1}, \ldots, \tau_{n}\right) ; \text { void }\right)
\end{aligned}
$$




$$
\begin{aligned}
& \Gamma, \Gamma_{1}, \ldots, \Gamma_{i-1} \vdash I_{i}:\left(\Psi_{i-1} ; \overrightarrow{F r}_{i-1}\right) \multimap \\
& \exists \Gamma_{i} .\left(\Psi_{i} ; \overrightarrow{F r}_{i} ; \mu_{i}\right) \quad \forall i \in 1 . . n \\
& n=\operatorname{fields}(C) \quad \ell \notin \operatorname{Dom}\left(\Gamma, \Gamma_{1}, \ldots, \Gamma_{n}\right) \quad \epsilon \in \operatorname{pre}(\operatorname{policy}(C)) \\
& \Gamma \vdash I_{1} \ldots I_{n} \text { newobj } C\left(\mu_{1}, \ldots, \mu_{n}\right) \text { : } \\
& \left(\Psi_{0} ; \overrightarrow{F r}_{0}\right) \multimap \exists \Gamma_{1}, \ldots, \Gamma_{n}, \ell: C .\left(\Psi_{n} \star(\ell \mapsto \epsilon) ; \overrightarrow{F r}_{n} ; C\langle\ell\rangle\right) \\
& \Gamma_{0}, \ldots, \Gamma_{j} \vdash I_{j}:\left(\Psi_{j}, \overrightarrow{F r}_{j}\right) \multimap \exists \Gamma_{j+1} .\left(\Psi_{j+1}, \overrightarrow{F r}_{j+1}, \tau_{j}\right) \forall j \in 0 . . n \\
& \tau_{0}=C\langle\ell\rangle \quad \ell \in \operatorname{Dom}\left(\Psi_{n+1}\right) \quad C:: \text { m.Sig } \in \operatorname{Dom}(\text { methodbody }) \\
& \Gamma_{0}, \ldots, \Gamma_{n} \vdash \operatorname{Sig}<:\left(\Psi_{\text {in }},\left(\tau_{0}, \ldots, \tau_{n}\right)\right) \multimap \exists \Gamma_{\text {out }} .\left(\Psi_{\text {out }}, F r_{\text {out }}, \tau\right) \\
& \Psi_{n+1}=\Psi_{\text {unused }} \star \Psi_{\text {in }} \\
& \Gamma_{0} \vdash I_{0} \ldots I_{n} \text { callvirt } C:: \text { m.Sig : } \\
& \left(\Psi_{0}, \overrightarrow{F r}_{0}\right) \multimap \exists \Gamma_{1}, \ldots, \Gamma_{n+1}, \Gamma_{\text {out }} .\left(\Psi_{\text {unused }} \star \Psi_{\text {out }}, \overrightarrow{F r}_{n+1}, \tau\right) \\
& H e \subseteq \operatorname{pre}(\operatorname{policy}(C)) \\
& \Gamma \vdash I:(\Psi ; \overrightarrow{F r}) \multimap \exists \Gamma^{\prime} .\left(\Psi^{\prime} \star(\ell \mapsto H) ; \overrightarrow{F r}^{\prime} ; C\langle\ell\rangle\right) \\
& \overrightarrow{\Gamma \vdash I \text { evt } e:(\Psi ; \overrightarrow{F r}) \multimap \exists \Gamma^{\prime} .\left(\Psi^{\prime} \star(\ell \mapsto H e) ; \overrightarrow{F r}^{\prime} ; \text { void }\right)} \\
& \ell \notin \operatorname{Dom}(\Gamma) \\
& \overline{\Gamma \vdash \text { newpackage } C:(\Psi ; \overrightarrow{F r}) \multimap \exists \ell: C\langle ?\rangle .(\Psi ; \overrightarrow{F r} ; C\langle ?\rangle)} \\
& H \subseteq H^{\prime} \subseteq \operatorname{policy}(C) \\
& \Gamma \vdash I_{1}:(\Psi ; \overrightarrow{F r}) \multimap \exists \Gamma_{1} .\left(\Psi_{1} ; \overrightarrow{F r}_{1} ; C\langle ?\rangle\right) \\
& \Gamma, \Gamma_{1} \vdash I_{2}:\left(\Psi_{1} ; \overrightarrow{F r}_{1}\right) \multimap \exists \Gamma_{2} .\left(\Psi_{2} ; \overrightarrow{F r}_{2} ; C\langle\ell\rangle\right) \\
& \underline{\Gamma, \Gamma_{1}, \Gamma_{2} \vdash I_{3}:\left(\Psi_{2} ; \overrightarrow{F r}_{2}\right) \multimap \exists \Gamma_{3} .\left(\Psi^{\prime} \star(\ell \mapsto H) ; \overrightarrow{F r}^{\prime} ; \operatorname{Rep}_{C}\left\langle H^{\prime}\right\rangle\right)} \\
& \Gamma \vdash I_{1} I_{2} I_{3} \text { pack : }(\Psi ; \overrightarrow{F r}) \multimap \exists \Gamma_{1}, \Gamma_{2}, \Gamma_{3} \cdot\left(\Psi^{\prime} ; \overrightarrow{F r}^{\prime} ; \text { void }\right) \\
& \ell \notin \operatorname{Dom}\left(\Psi^{\prime}\right) \quad \theta \notin \operatorname{Dom}(\Gamma) \\
& \Gamma \vdash I:(\Psi ; \overrightarrow{F r}) \multimap \exists \Gamma^{\prime} .\left(\Psi^{\prime} ; \overrightarrow{F r}^{\prime}\left(\tau_{0}, \ldots, \tau_{n}\right) ; C\langle ?\rangle\right) \\
& \Gamma \vdash I \text { unpack } j:(\Psi ; \overrightarrow{F r}) \multimap \exists \Gamma^{\prime}, \ell: C, \theta \text {. } \\
& \left(\Psi^{\prime}, \ell \mapsto \theta ; \overrightarrow{\operatorname{Fr}}^{\prime}\left(\tau_{0}, \ldots, \tau_{j-1}, \operatorname{Rep}_{C}\langle\theta\rangle, \tau_{j+1}, \ldots, \tau_{n}\right) ; C\langle\ell\rangle\right) \\
& \Gamma \vdash I_{1}:(\Psi ; \overrightarrow{F r}) \multimap \exists \Gamma_{1} .\left(\Psi_{1} ; \overrightarrow{F r}_{1} ; \operatorname{Rep}_{C}\langle H\rangle\right) \\
& \Gamma, \Gamma_{1} \vdash I_{2}:\left(c t x_{C, k}^{+}\left(H, \Psi_{1}\right) ; \overrightarrow{F r}_{1}\right) \multimap \exists \Gamma^{\prime} .\left(\Psi^{\prime} ; \overrightarrow{F r}^{\prime} ; \tau\right) \\
& \Gamma, \Gamma_{1} \vdash I_{3}:\left(c t x_{C, k}^{-}\left(H, \Psi_{1}\right) ; \overrightarrow{F r}_{1}\right) \multimap \exists \Gamma^{\prime} .\left(\Psi^{\prime} ; \overrightarrow{F r}^{\prime} ; \tau\right) \\
& \Gamma \vdash I_{1} I_{2} I_{3} \text { condst } k:(\Psi ; \overrightarrow{F r}) \multimap \exists \Gamma_{1}, \Gamma^{\prime} .\left(\Psi^{\prime} ; \overrightarrow{F r}^{\prime} ; \tau\right) \\
& \Gamma \vdash I_{i}:\left(\Psi_{i-1} ; \overrightarrow{F r}_{i-1}\right) \multimap \exists \Gamma_{i} .\left(\Psi_{i} ; \overrightarrow{F r}_{i} ; \operatorname{Rep}_{C_{i}}\left\langle H_{i}\right\rangle\right) \forall i \in 1 . . n \\
& \Gamma \vdash I_{1} \ldots I_{n} \text { newhist } C, k:\left(\Psi_{0} ; \overrightarrow{F r}_{0}\right) \multimap \exists \Gamma_{1}, \ldots, \Gamma_{n} \text {. } \\
& \left(\Psi_{n} ; \overrightarrow{F r}_{n} ; H C_{C, k}\left(\operatorname{Rep}_{C_{1}}\left\langle H_{1}\right\rangle, \ldots, \operatorname{Rep}_{C_{n}}\left\langle H_{n}\right\rangle\right)\right) \\
& \Gamma_{1}, \Gamma^{\prime} \vdash I:\left(\Psi_{1} ; \overrightarrow{F r}_{1}\right) \multimap \exists \Gamma_{2} \cdot\left(\Psi_{2} ; \overrightarrow{F r}_{2} ; \tau\right) \\
& \Psi_{1}^{\prime} \preceq \Psi_{1} \quad \overrightarrow{F r}_{1}^{\prime} \preceq \overrightarrow{F r}_{1} \quad \Psi_{2} \preceq \Psi_{2}^{\prime} \quad \overrightarrow{F r}_{2} \preceq \overrightarrow{F r}_{2}^{\prime} \quad \tau \preceq \tau^{\prime} \\
& \Gamma_{1}, \Gamma^{\prime} \vdash I:\left(\Psi_{1}^{\prime} ; \overrightarrow{F r}_{1}^{\prime}\right) \multimap \exists \Gamma_{2}, \Gamma^{\prime} .\left(\Psi_{2}^{\prime} ; \overrightarrow{F r}_{2}^{\prime} ; \tau^{\prime}\right) \\
& \overrightarrow{\Gamma \vdash \mathbf{0}:(\Psi ; \overrightarrow{F r}) \multimap(\Psi ; \overrightarrow{F r} ; \text { void })} \\
& \Gamma \vdash i 4:(\Psi ; \overrightarrow{F r}) \multimap(\Psi ; \overrightarrow{F r} ; \text { int32 }) \\
& \frac{\Psi=\Psi^{\prime} \star(\ell \mapsto H)}{\Gamma, \ell: C \vdash \ell:(\Psi ; \overrightarrow{F r}) \multimap(\Psi ; \overrightarrow{F r} ; C\langle\ell\rangle)}
\end{aligned}
$$




$$
\begin{gathered}
\overline{\Gamma, \ell: C\langle ?\rangle \vdash \ell:(\Psi ; \overrightarrow{F r}) \multimap(\Psi ; \overrightarrow{F r} ; C\langle ?\rangle)} \\
\hline \Gamma \vdash \overrightarrow{\operatorname{rep} p_{C}(H)}:(\Psi ; \overrightarrow{F r}) \multimap\left(\Psi ; \overrightarrow{F r} ; \operatorname{Rep}_{C}\langle H\rangle\right) \\
\frac{\Gamma \vdash I:(\Psi ; \overrightarrow{F r}) \multimap \exists \Gamma^{\prime} .\left(\Psi^{\prime} ; \overrightarrow{F r}^{\prime} F r_{0} ; \tau\right)}{\Gamma \vdash I \text { ret }:(\Psi ; \overrightarrow{F r}) \multimap \exists \Gamma^{\prime} .\left(\Psi^{\prime} ; \overrightarrow{F r}^{\prime} ; \tau\right)}
\end{gathered}
$$

The judgment $\Gamma \vdash \operatorname{Sig}_{1}<: \operatorname{Sig}_{2}$ in rule 45 asserts that $\operatorname{Sig}_{1}$ alphavaries to $\mathrm{Sig}_{2}$. That is, there exists a substitution $\sigma: \ell \rightarrow \ell$ such that $\sigma\left(\operatorname{Sig}_{1}\right)=\operatorname{Sig}_{2}$ and any free variables in $\operatorname{Sig}_{2}$ are drawn from $\Gamma$. This captures the requirement that call sites must satisfy the callee's precondition and can assume the callee's postcondition.

\section{B Typing Proofs}

The proofs of Terminating Policy Adherence (Theorem 1) and of Nonterminating Prefix Adherence (Theorem 2 ) are arrived at in three steps. First, in 8.2 we prove subject reduction for the type system. That is, we prove that taking a step according to the operational semantics provided in Figure 7preserves the type of a Mobile term as defined in Appendix A. Second, in $\$ \mathrm{~B} .3$ we prove that well-typed Mobile terms can take a step as long as they have not been reduced to a value or have not entered a "bad" state, such as by performing an unpack operation on an empty package. Third, these two results are leveraged in $\$ \widehat{B} .4$ to prove Terminating Policy Adherence and Non-terminating Prefix Adherence theorems. That is, we show that well-typed Mobile programs that terminate normally will satisfy the security policy, and that well-typed Mobile programs that do not terminate or that enter a "bad" state will satisfy a prefix of the security policy.

\section{B.1 Canonical Derivations}

In the proofs that follow, it will be useful to appeal to the following "obvious" facts about the derivation system given in Figure 9. (Proofs of the facts below can be obtained by trivial inductions over the derivations of the various relevant judgments.)

Fact 1. If $\Gamma^{\prime} \vdash_{\text {heap }} h: \Gamma$ holds then the following three statements are equivalent:

(i) $\Gamma=\Gamma_{0}, \ell: C$

(ii) $h=h_{0},\left(\ell \mapsto o b j_{C}\left\{f_{i}=v_{i} \mid i \in 1 \text {...fields }(C)\right\}^{\vec{e}}\right)$

(iii) There exists a derivation of $\Gamma \vdash_{\text {heap }} h: \Gamma$ that ends in

$$
\frac{\Gamma^{\prime} \vdash v_{i}:(\Psi ; \overrightarrow{F r}) \multimap\left(\Psi ; \overrightarrow{F r} ; \text { field }\left(C, f_{i}\right)\right) \forall i \in 1 . \text { fields }(C)}{\Gamma^{\prime} \vdash_{\text {heap }} h: \Gamma}
$$


and the following three statements are equivalent:

(i) $\Gamma=\Gamma_{0}, \ell: C\langle ?\rangle$

(ii) $h=h_{0},(\ell \mapsto p k g(\ldots))$

(iii) There exists a derivation of $\Gamma \vdash_{\text {heap }} h: \Gamma$ that ends in

$$
\frac{\Gamma^{\prime} \vdash_{\text {heap }} h_{0}: \Gamma_{0}}{\Gamma^{\prime} \vdash_{\text {heap }} h: \Gamma}
$$

Fact 2. If $\vdash_{\text {hist }} h:(\Gamma ; \Psi)$ holds then the following three statements are equivalent:

(i) $\Gamma=\Gamma_{0}, \ell^{\prime}: C$

(ii) $h=h_{0},\left(\ell^{\prime} \mapsto o b j_{C}\{\ldots\}^{\vec{e}}\right)$

(iii) There exists a derivation of $\vdash_{h i s t} h:(\Gamma ; \Psi)$ that ends in one of

$$
\begin{gathered}
\frac{\vdash_{h i s t} h_{0}:\left(\Gamma_{0} ; \Psi_{0}\right) \quad \vec{e} \subseteq H}{\vdash_{h i s t} h:(\Gamma ; \Psi)}(28), \\
\frac{\vdash_{h i s t} h_{1}:\left(\Gamma_{1} ; \Psi\right) \quad \vec{e} \subseteq H \subseteq \operatorname{policy}(C)}{\vdash_{h i s t} h:(\Gamma ; \Psi)} \\
\frac{\vdash_{\text {hist }} h_{0}:\left(\Gamma_{0} ; \Psi\right) \quad \vec{e} \subseteq \operatorname{policy}(C)}{\vdash_{h i s t} h:(\Gamma ; \Psi)}
\end{gathered}
$$

where $\Psi=\Psi_{0} \star\left(\ell^{\prime} \mapsto H\right), \Gamma_{1}=\Gamma_{0}, \ell: C\langle ?\rangle$, and $h_{1}=h_{0},(\ell \mapsto$ $\left.\operatorname{pkg}\left(\ell^{\prime}, \operatorname{rep}_{C}(H)\right)\right)$;

and the following three statements are equivalent:

(i) $\Gamma=\Gamma_{0}, \ell: C\langle ?\rangle$

(ii) $h=h_{0},(\ell \mapsto p k g(\ldots))$

(iii) There exists a derivation of $\vdash_{\text {hist }} h:(\Gamma ; \Psi)$ that ends in one of

$$
\frac{\vdash_{\text {hist }} h_{1}:\left(\Gamma_{1} ; \Psi\right) \quad \vec{e} \subseteq H \subseteq \operatorname{policy}(C)}{\vdash_{\text {hist }} h:(\Gamma ; \Psi)}(29)
$$

or

$$
\frac{\vdash_{\text {hist }} h_{0}:\left(\Gamma_{0} ; \Psi\right)}{\vdash_{\text {hist }} h:(\Gamma ; \Psi)}
$$

where $\Gamma_{1}=\Gamma_{0}, \ell: C\langle ?\rangle$ and $h_{1}=h_{0},\left(\ell \mapsto p k g\left(\ell^{\prime}, \operatorname{rep}_{C}(H)\right)\right)$.

Fact 3. The following judgments can be weakened in the following ways:

1. If $\Gamma_{0} \vdash_{\text {heap }} h: \Gamma$ holds then $\Gamma_{0}, \Gamma^{\prime} \vdash_{\text {heap }} h: \Gamma$ also holds. 
2. If $\Gamma_{0} \vdash_{\text {stack }} s: \overrightarrow{F r}$ holds then $\Gamma_{0}, \Gamma^{\prime} \vdash_{\text {stack }} s: \overrightarrow{F r}$ also holds.

3. If $\Gamma_{0} \vdash I:(\Psi ; \overrightarrow{F r}) \multimap \exists \Gamma^{\prime \prime} .\left(\Psi^{\prime \prime} ; \overrightarrow{F r}^{\prime \prime} ; \tau\right)$ holds then $\Gamma_{0}, \Gamma^{\prime} \vdash I$ : $(\Psi ; \overrightarrow{F r}) \multimap \exists \Gamma^{\prime \prime} .\left(\Psi^{\prime \prime} ; \overrightarrow{F r}^{\prime \prime} ; \tau\right)$ also holds.

Facts 1 and 2 state that when $\Gamma^{\prime} \vdash_{\text {heap }} h: \Gamma$ holds or $\vdash_{\text {hist }} h:(\Gamma ; \Psi)$ holds, then $\Gamma$ and $h$ match element for element, and there is a way to reorganize the derivation of either judgment to bring the rule that refers to any particular element to the bottom of the derivation tree. That is, the rule applications in either derivation can be reordered arbitrarily. Fact 3 states that judgment $\Gamma_{0} \vdash_{\text {heap }} h: \Gamma$, judgment $\Gamma_{0} \vdash_{\text {stack }} s: \overrightarrow{F r}$, and judgment $\Gamma_{0} \vdash I:(\Psi ; \overrightarrow{F r}) \multimap \exists \Gamma^{\prime \prime} .\left(\Psi^{\prime \prime} ; \overrightarrow{F r}^{\prime \prime} ; \tau\right)$ can be weakened by adding more elements to $\Gamma_{0}$.

\section{B.2 Subject Reduction}

Lemma 1 (Context Widening). If $\Gamma \vdash I:(\Psi ; F r) \multimap \exists \Gamma^{\prime} .\left(\Psi^{\prime} ; F r^{\prime} ; \tau\right)$ holds and $I$ contains no ret instructions, then $\Gamma \vdash I:\left(\Psi_{\text {extra }} \star \Psi ; \overrightarrow{F r} F r\right) \multimap$ $\exists \Gamma^{\prime} .\left(\Psi_{\text {extra }} \star \Psi^{\prime} ; \overrightarrow{F r} F r^{\prime} ; \tau\right)$ holds.

Proof. Observe that all typing rules except the typing rule for ret (58) are parameterized by an arbitrary frame list prefix that remains unchanged by an application of the rule. Since $I$ has no ret instructions, this suffices to prove that $\Gamma \vdash I:(\Psi ; \overrightarrow{F r} F r) \multimap \exists \Gamma^{\prime} .\left(\Psi^{\prime} ; \overrightarrow{F r} F r^{\prime} ; \tau\right)$ holds.

It remains to show that $\Psi \vdash I:\left(\Psi_{\text {extra }} \star \Psi ; F r\right) \multimap \exists \Gamma^{\prime} .\left(\Psi_{\text {extra }} \star \Psi^{\prime} ; F r^{\prime} ; \tau\right)$ holds. Let $\mathcal{D}$ be the derivation of $\Gamma \vdash I:(\Psi ; F r) \multimap \exists \Gamma^{\prime} .\left(\Psi^{\prime} ; F r^{\prime} ; \tau\right)$. Proof is by induction on the structure of $\mathcal{D}$.

Case 1: $\mathcal{D}$ ends in rule 36, 42, 47, 53, 54, 55, 56, or 57, In these cases, $\Psi^{\prime}=\Psi$. The lemma follows immediately by instantiating $\Psi$ with $\Psi_{\text {extra }} \star \Psi$ in each typing rule.

Case 2: $\mathcal{D}$ ends in rule 37, 38, 39, 40, 41, 43, 44, 46, 48, 49, 50, or 51. The lemma follows by inductive hypothesis, by instantiating each antecedent of the form $\Gamma_{0} \vdash I_{0}:\left(\Psi_{0} ; F r_{0}\right) \multimap \exists \Gamma_{0}^{\prime} .\left(\Psi_{0}^{\prime} ; F r_{0}^{\prime} ; \tau_{0}\right)$ with $\Gamma_{0} \vdash I_{0}:\left(\Psi_{\text {extra }} \star \Psi_{0} ; F r_{0}\right) \multimap \exists \Gamma_{0}^{\prime} .\left(\Psi_{\text {extra }} \star \Psi_{0}^{\prime} ; F r_{0}^{\prime} ; \tau_{0}\right)$.

Case 3: $\mathcal{D}$ ends in rule 45. In addition to instantiating into each antecedent as in the previous case, instantiate $\Psi_{\text {unused }}$ with $\Psi_{\text {extra }} \star \Psi_{\text {unused }}$. The lemma then holds by inductive hypothesis.

Case 4: $\mathcal{D}$ ends in rule 52 . Observe from the subtyping rules that if $\Psi_{1} \preceq$ $\Psi_{1}^{\prime}$ then $\Psi_{\text {extra }} \star \Psi_{1} \preceq \Psi_{\text {extra }} \star \Psi_{1}^{\prime}$. We can therefore instantiate the rule's antecedents as in the previous two cases to prove the lemma by inductive hypothesis. 
Lemma 2 (Context Subtyping). If $\vdash_{\text {hist }} h:(\Gamma ; \Psi)$ and $\Psi \preceq \Psi^{\prime}$ hold then $\vdash_{\text {hist }} h:\left(\Gamma ; \Psi^{\prime}\right)$ holds.

Proof. Let $\mathcal{D}$ be a derivation of $\vdash_{\text {hist }} h:(\Gamma ; \Psi)$. Proof is by induction over the structure of $\mathcal{D}$.

Base Case: If $\mathcal{D}$ ends with rule 33 , then $\Psi=\Psi^{\prime}=\cdot$ and the lemma holds immediately.

Inductive Case: If $\mathcal{D}$ ends in any remaining rule other than rule 28 , then the lemma follows immediately from the inductive hypothesis. Assume $\mathcal{D}$ ends in rule 28 and therefore has the form

$$
\frac{\vdash_{\text {hist }} h_{0}:\left(\Gamma_{0} ; \Psi_{0}\right) \quad \vec{e} \subseteq H}{\vdash_{\text {hist }} h:(\Gamma ; \Psi)}(28)
$$

where $\Gamma=\Gamma_{0}, \ell: C, h=h_{0},\left(\ell \mapsto o b j_{C}\{\ldots\}^{\vec{e}}\right)$, and $\Psi=\Psi_{0} \star(\ell \mapsto H)$. Since $\Psi \preceq \Psi^{\prime}$, it follows that $\Psi^{\prime}=\Psi_{0}^{\prime} \star\left(\ell \mapsto H^{\prime}\right)$ such that $H \subseteq H^{\prime}$ and $\Psi_{0} \preceq \Psi_{0}^{\prime}$. Thus, by inductive hypothesis one can derive

$$
\frac{\vdash_{h i s t} h_{0}:\left(\Gamma_{0} ; \Psi_{0}^{\prime}\right) \quad \vec{e} \subseteq H^{\prime}}{\vdash_{\text {hist }} h:\left(\Gamma ; \Psi^{\prime}\right)} 28
$$

Lemma 3 (Stepwise Subject Reduction). Assume that

$$
\begin{gathered}
\Gamma \vdash \psi:(\Psi ; \overrightarrow{F r}) \\
\Gamma \vdash I:(\Psi ; \overrightarrow{F r}) \multimap \exists \Gamma^{\prime \prime} .\left(\Psi^{\prime \prime} ; \overrightarrow{F r}^{\prime \prime} ; \tau\right)
\end{gathered}
$$

both hold and assume that all methods in Dom(methodbody), are well-typed. If $\psi, I \rightsquigarrow \psi^{\prime}, I^{\prime}$ holds then there exists $\Gamma^{\prime}, \Psi^{\prime}, \overrightarrow{F r}^{\prime}$, and $\sigma: \theta \rightarrow \vec{e}$ such that $\Gamma^{\prime} \vdash \psi^{\prime}:\left(\Psi^{\prime} ; \overrightarrow{F r}^{\prime}\right)$ holds and $\Gamma^{\prime} \vdash I^{\prime}:\left(\Psi^{\prime} ; \overrightarrow{F r}^{\prime}\right) \multimap \exists \Gamma^{\prime \prime} .\left(\sigma\left(\Psi^{\prime \prime}\right) ; \sigma\left(\overrightarrow{F r}^{\prime \prime}\right) ; \sigma(\tau)\right)$ holds.

Proof. Proof is by induction on the derivation of the judgment $\psi, I \rightsquigarrow \psi^{\prime}, I^{\prime}$. To make the proof more tractable, in what follows we make the simplifying assumption that weakening rule 52 does not appear in the derivation of judgment 60. Similar logic to that presented below applies to cases where rule 52 is present.

Case 1: $\psi$, ldc.i4 $i 4 \rightsquigarrow \psi, i 4$. Then $\Gamma^{\prime \prime}=\cdot$ and $\left(\Psi^{\prime \prime} ; \overrightarrow{F r} ; \tau\right)=(\Psi ; \overrightarrow{F r} ; \operatorname{int32})$ by 36. To satisfy the lemma, choose $\Gamma^{\prime}=\Gamma, \Psi^{\prime}=\Psi, \overrightarrow{F r}^{\prime}=\overrightarrow{F r}$, and $\sigma=\cdot$ and apply typing rule 54 . 
Case 2: $\psi, E\left[I_{0}\right] \rightsquigarrow \psi^{\prime}, E\left[I_{0}^{\prime}\right]$. Let $\mathcal{D}$ be a derivation of 60 . Observe that for all possible $E\left[I_{0}\right]$, derivation $\mathcal{D}$ includes a subderivation $\mathcal{D}_{2}$ of $\Gamma \vdash I_{0}:(\Psi ; \overrightarrow{F r}) \multimap \exists \Gamma_{2}^{\prime} .\left(\Psi_{2}^{\prime} ; \overrightarrow{F r}_{2}^{\prime} ; \tau_{2}\right)$. By inductive hypothesis, there exists $\Gamma^{\prime}, \Psi^{\prime}, \overrightarrow{F r}^{\prime}$, and $\sigma$ such that $\Gamma^{\prime} \vdash \psi^{\prime}:\left(\Psi^{\prime} ; \overrightarrow{F r}^{\prime}\right)$ and $\Gamma^{\prime} \vdash$ $I_{0}^{\prime}:\left(\Psi^{\prime} ; \overrightarrow{F r}^{\prime}\right) \multimap \exists \Gamma_{2}^{\prime} .\left(\sigma\left(\Psi_{2}^{\prime}\right) ; \sigma\left(\overrightarrow{F r}_{2}^{\prime}\right) ; \sigma\left(\tau_{2}\right)\right)$. Let $\mathcal{D}_{2}^{\prime}$ be a derivation of this latter judgment. Then derivation $\mathcal{D}$ can be modified by replacing subderivation $\mathcal{D}_{2}$ with derivation $\mathcal{D}_{2}^{\prime}$ to obtain a derivation of $\Gamma^{\prime} \vdash E\left[I_{0}^{\prime}\right]:\left(\Psi^{\prime} ; \overrightarrow{F r}^{\prime}\right) \multimap \exists \Gamma^{\prime \prime} .\left(\sigma\left(\Psi^{\prime \prime}\right) ; \sigma\left(\overrightarrow{F r}^{\prime \prime}\right) ; \sigma(\tau)\right)$.

Case 3: $\psi, i 4 I_{2} I_{3}$ cond $\rightsquigarrow \psi, I_{j}$ where $j \in\{2,3\}$. Any derivation of 60 contains a subderivation of $\Gamma \vdash I_{j}:(\Psi ; \overrightarrow{F r}) \multimap \exists \Gamma^{\prime \prime} .\left(\Psi^{\prime \prime} ; \overrightarrow{F r}^{\prime \prime} ; \tau\right)$ (by 37 and 54 . Thus the lemma is satisfied by choosing $\Gamma^{\prime}=\Gamma, \Psi^{\prime}=\Psi$, $\overrightarrow{F r}^{\prime}=\overrightarrow{F r}$, and $\sigma=$.

Case 4: $\psi, I_{1} I_{2}$ while $\rightsquigarrow \psi, I_{1}\left(I_{2} ;\left(I_{1} I_{2}\right.\right.$ while $\left.)\right) 0$ cond. Any derivation of 60 must have the form

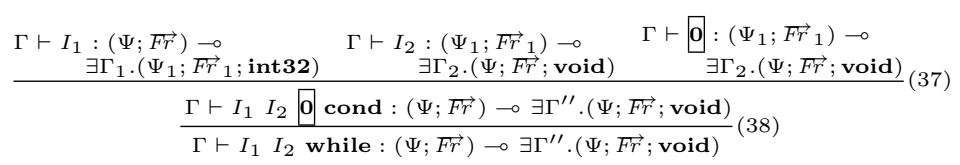

where $\Gamma=\Gamma_{0}, \Gamma^{\prime \prime}$ and $\Gamma^{\prime \prime}=\Gamma_{1}, \Gamma_{2}$. One can therefore derive

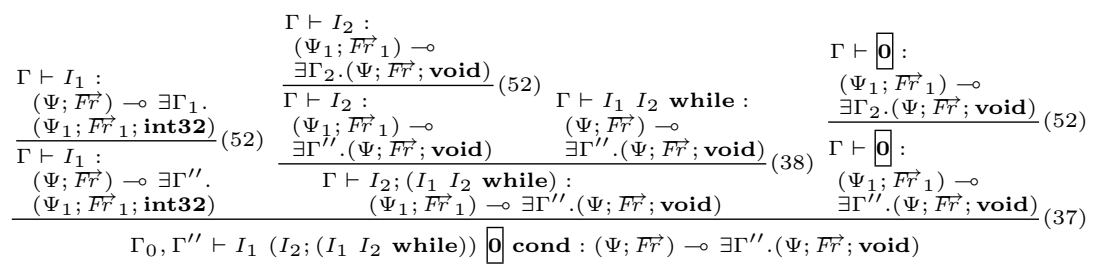

The lemma is thus satisfied by choosing $\Gamma^{\prime}=\Gamma, \Psi^{\prime}=\Psi, \overrightarrow{F r}^{\prime}=\overrightarrow{F r}$, and $\sigma=$.

Case 5: $\psi, v ; I_{2} \rightsquigarrow \psi, I_{2}$. Any derivation of 60 contains a subderivation of $\Gamma \vdash I_{2}:(\Psi ; \overrightarrow{F r}) \multimap \exists \Gamma^{\prime \prime} .\left(\Psi^{\prime \prime} ; \overrightarrow{F r}^{\prime \prime} ; \tau\right)$ (by 39 and 53 ). Thus the lemma is satisfied by choosing $\Gamma^{\prime}=\Gamma, \Psi^{\prime}=\Psi, \overrightarrow{F r}=\overrightarrow{F r}$, and $\sigma=\cdot$.

Case 6: $\psi$, ldarg $j \rightsquigarrow \psi, v_{j}$ where $\psi=\left(h, s\left(v_{0}, \ldots, v_{n}\right)\right)$. From 60 and 42 , $\overrightarrow{F r}$ has the form $\overrightarrow{F r}_{0} F r$ and $0 \leq j \leq n$. From 59 and 34 , $F r=$ $\left(\tau_{0}, \ldots, \tau_{n}\right)$ and $\Gamma \vdash v_{j}:(\Psi ; \overrightarrow{F r}) \multimap\left(\Psi ; \overrightarrow{F r} ; \tau_{j}\right)$ holds. The lemma is therefore satisfied by choosing $\Gamma^{\prime}=\Gamma, \Psi^{\prime}=\Psi, \overrightarrow{F r}^{\prime}=\overrightarrow{F r}$, and $\sigma=\cdot$.

Case 7: $(h, s), v$ starg $j \rightsquigarrow\left(h, s^{\prime}\right), 0$ where $s=s_{0}\left(v_{0}, \ldots, v_{n}\right)$ for some stack prefix $s_{0}$, and $s^{\prime}=s_{0}\left(v_{0}, \ldots, v_{j-1}, v, v_{j+1}, \ldots, v_{n}\right)$. From 60 and $43, \overrightarrow{F r}$ has the form $\overrightarrow{F r}_{0} F r$, and $0 \leq j \leq n$. From 59 and 34, $F r=\left(\tau_{0}, \ldots, \tau_{n}\right)$. From 60 and $43, \Gamma^{\prime \prime}=\cdot \Psi^{\prime \prime}=\Psi$, 
$\overrightarrow{F r}^{\prime \prime}=\overrightarrow{F r}_{0}\left(\tau_{0}, \ldots, \tau_{j-1}, \tau^{\prime}, \tau_{j+1}, \ldots, \tau_{n}\right), \tau=$ void, and $\Gamma \vdash v$ : $(\Psi ; \overrightarrow{F r}) \multimap\left(\Psi ; \overrightarrow{F r} ; \tau^{\prime}\right)$ holds. Choose $\Gamma^{\prime}=\Gamma, \Psi^{\prime}=\Psi, \overrightarrow{F r}^{\prime}=\overrightarrow{F r}^{\prime \prime}$, and $\sigma=\cdot$. Since all type judgments for value expressions are independent of frames, one can derive $\Gamma \vdash v:\left(\Psi^{\prime} ; \overrightarrow{F r}^{\prime}\right) \multimap\left(\Psi^{\prime} ; \overrightarrow{F r}^{\prime} ; \tau^{\prime}\right)$ to prove by 34 that $\Gamma^{\prime} \vdash\left(h ; s^{\prime}\right):\left(\Psi^{\prime} ; \overrightarrow{F r}^{\prime}\right)$ holds. Furthermore, $\Gamma^{\prime} \vdash \mathbf{0}:\left(\Psi^{\prime} ; \overrightarrow{F r}^{\prime}\right) \multimap\left(\Psi^{\prime \prime} ; \overrightarrow{F r}^{\prime \prime} ; \tau\right)$ holds by 53 , satisfying the lemma.

Case 8: $(h, s), v_{1} \ldots v_{n}$ newobj $C\left(\mu_{1}, \ldots, \mu_{n}\right) \rightsquigarrow\left(h^{\prime}, s\right)$, $\ell$ where $h^{\prime}=$ $h,\left(\ell \mapsto o b j_{C}\left\{f_{i}=v_{i} \mid i \in 1 . . n\right\}^{\epsilon}\right)$ and $n=$ fields $(C)$. From 60 and 44 , $\Gamma^{\prime \prime}=\ell: C, \Psi^{\prime \prime}=\Psi \star(\ell \mapsto \epsilon), \overrightarrow{F r}^{\prime \prime}=\overrightarrow{F r}$, and $\tau=C\langle\ell\rangle$. Additionally, $\Gamma \vdash v_{i}:(\Psi ; \overrightarrow{F r}) \multimap\left(\Psi ; \overrightarrow{F r} ; \operatorname{field}\left(C, f_{i}\right)\right) \forall i \in 1 . . n$. Choose $\Gamma^{\prime}=\Gamma^{\prime \prime}$, $\Psi^{\prime}=\Psi^{\prime \prime}, \overrightarrow{F r}^{\prime}=\overrightarrow{F r}^{\prime \prime}$, and $\sigma=\cdot$. From 59 one can derive

$\frac{\Gamma^{\prime} \vdash_{\text {heap }} h: \Gamma \quad \Gamma^{\prime} \vdash v_{i}:(\Psi ; \overrightarrow{F r}) \multimap\left(\Psi ; \overrightarrow{F r} ; \text { field }\left(C, f_{i}\right)\right) \forall i \in 1 . . n}{\Gamma^{\prime} \vdash_{\text {heap }} h^{\prime}: \Gamma^{\prime}}$

and derive

$$
\frac{\vdash_{\text {hist }} h:\left(\Gamma^{\prime} ; \Psi\right) \quad \epsilon \subseteq \epsilon}{\vdash_{\text {hist }} h^{\prime}:\left(\Gamma^{\prime} ; \Psi^{\prime}\right)} 24
$$

Thus $\Gamma^{\prime} \vdash\left(h^{\prime}, s\right):\left(\Psi^{\prime}, \overrightarrow{F r}^{\prime}\right)$ holds. Further, observe that $\Gamma^{\prime} \vdash \ell$ : $\left(\Psi^{\prime} ; \overrightarrow{F r}^{\prime}\right) \multimap \exists \Gamma^{\prime \prime} .\left(\Psi^{\prime \prime} ; F r^{\prime \prime} ; C\langle\ell\rangle\right)$ holds by 55 because $\Gamma^{\prime}=\Gamma, \ell: C$. Thus the lemma is satisfied.

Case 9: $(h, s), v_{0} \ldots v_{n}$ callvirt $C:: m . S i g \rightsquigarrow(h, s a), I_{0}$ ret where $a=$ $\left(v_{0}, \ldots, v_{n}\right)$ and $I_{0}=$ methodbody $(C::$ m.Sig $)$. From 60 and $45, \overrightarrow{F r}^{\prime \prime}=$ $\overrightarrow{F r}$, and there exists $\left(\Psi_{\text {in }},\left(\tau_{0}, \ldots, \tau_{n}\right)\right), \Psi_{\text {out }}, \Psi_{\text {unused }}$, and $\overrightarrow{F r}_{\text {out }}$ such that $\Psi=\Psi_{\text {unused }} \star \Psi_{\text {in }}, \Psi^{\prime \prime}=\Psi_{\text {unused }} \star \Psi_{\text {out }}$,

$$
\Gamma \vdash \overrightarrow{v_{i}}:(\Psi ; \overrightarrow{F r}) \multimap\left(\Psi ; \overrightarrow{F r} ; \tau_{i}\right) \forall i \in 0 . . n
$$

and

$$
\Gamma \vdash \operatorname{Sig}<:\left(\Psi_{\text {in }} ;\left(\tau_{0}, \ldots, \tau_{n}\right)\right) \multimap \exists \Gamma^{\prime \prime} .\left(\Psi_{\text {out }} ; F r_{\text {out }} ; \tau\right) .
$$

Since $C:: m . S i g$ is well-typed, it follows that

$$
\Gamma \vdash I_{0}:\left(\Psi_{\text {in }} ;\left(\tau_{0}, \ldots, \tau_{n}\right)\right) \multimap \exists \Gamma^{\prime \prime} .\left(\Psi_{\text {out }} ; F r_{\text {out }} ; \tau\right)
$$

By context widening, this implies that

$$
\begin{array}{r}
\Gamma \vdash I_{0}:\left(\Psi_{\text {unused }} \star \Psi_{\text {in }} ; \overrightarrow{F r}\left(\tau_{0}, \ldots, \tau_{n}\right)\right) \multimap \\
\exists \Gamma^{\prime \prime} .\left(\Psi_{\text {unused }} \star \Psi_{\text {out }} ; \overrightarrow{F r} F r_{\text {out }} ; \tau\right)
\end{array}
$$


which collapses to

$$
\Gamma \vdash I_{0}:\left(\Psi ; \overrightarrow{F r}\left(\tau_{0}, \ldots, \tau_{n}\right)\right) \multimap \exists \Gamma^{\prime \prime} .\left(\Psi^{\prime \prime} ; \overrightarrow{F r} F r_{\text {out }} ; \tau\right)
$$

Choose $\Gamma^{\prime}=\Gamma, \Psi^{\prime}=\Psi, \overrightarrow{F r}^{\prime}=\overrightarrow{F r}\left(\tau_{0}, \ldots, \tau_{n}\right)$, and $\sigma=\cdot$. To prove that $\Gamma^{\prime} \vdash I^{\prime}:\left(\Psi^{\prime} ; \overrightarrow{F r}^{\prime}\right) \multimap \exists \Gamma^{\prime \prime} .\left(\Psi^{\prime \prime} ; \overrightarrow{F r}^{\prime \prime} ; \tau\right)$ holds, derive

$$
\frac{\Gamma \vdash I_{0}:\left(\Psi ; \overrightarrow{F r}\left(\tau_{0}, \ldots, \tau_{n}\right)\right) \multimap \exists \Gamma^{\prime \prime} .\left(\Psi^{\prime \prime} ; \overrightarrow{F r} F r_{\text {out }} ; \tau\right)}{\Gamma \vdash I_{0} \text { ret }:\left(\Psi ; \overrightarrow{F r}\left(\tau_{0}, \ldots, \tau_{n}\right)\right) \multimap \exists \Gamma^{\prime \prime} .\left(\Psi^{\prime \prime} ; \overrightarrow{F r} ; \tau\right)}
$$

(recalling that $\left.\overrightarrow{F r}^{\prime \prime}=\overrightarrow{F r}\right)$. To prove that $\Gamma^{\prime} \vdash(h ; s a):\left(\Psi^{\prime} ; \overrightarrow{F r}^{\prime}\right)$ holds, observe that $\Gamma \vdash_{\text {stack }} s: \overrightarrow{F r}$ holds by 59 , and therefore one can derive

$$
\frac{\Gamma \vdash_{\text {stack }} s: \overrightarrow{F r} \quad \Gamma \vdash v_{i}:(\Psi ; \overrightarrow{F r}) \multimap\left(\Psi ; \overrightarrow{F r} ; \tau_{i}\right) \forall i \in 0 . . n}{\Gamma^{\prime} \vdash_{\text {stack }} s a: \overrightarrow{F r}^{\prime}}
$$

by 61 and 34 .

Case 10: $(h, s a), v$ ret $\rightsquigarrow(h, s), v$. By 60 and $58, \Gamma^{\prime \prime}=\cdot, \Psi=\Psi^{\prime \prime}$, and $\overrightarrow{F r}=\overrightarrow{F r}^{\prime \prime} F r_{0}$ for some $F r_{0}$. Choose $\Gamma^{\prime}=\Gamma, \Psi^{\prime}=\Psi^{\prime \prime}, \overrightarrow{F r}^{\prime}=\overrightarrow{F r}^{\prime \prime}$, and $\sigma=\cdot$. Any derivation of 60 has a subderivation of $\Gamma \vdash v:(\Psi ; \overrightarrow{F r}) \multimap$ $(\Psi ; \overrightarrow{F r} ; \tau)$. Since the typing rules for value expressions are independant of frame, one can therefore derive $\Gamma \vdash v:\left(\Psi^{\prime} ; \overrightarrow{F r}^{\prime}\right) \multimap\left(\Psi^{\prime \prime} ; \overrightarrow{F r}^{\prime \prime} ; \tau\right)$. Furthermore, one derivation of 59 has a subderivation of

$$
\frac{\Gamma \vdash_{\text {stack }} s: \overrightarrow{F r}^{\prime \prime} \quad \vdots}{\Gamma \vdash_{s t a c k} s a: \overrightarrow{F r}^{\prime \prime} F_{0}}
$$

Hence $\Gamma^{\prime} \vdash_{\text {stack }} s: \overrightarrow{F r}^{\prime}$ holds.

Case 11: $(h, s)$, $\ell$ ldfld $\mu C:: f \rightsquigarrow(h, s), v$ where $h(\ell)=o b j_{C}\{\ldots, f=$ $v, \ldots\}^{\vec{e}}$. By 60 and $40, \Gamma^{\prime \prime}=\cdot, \Psi=\Psi^{\prime \prime}$, and $\overrightarrow{F r}=\overrightarrow{F r}^{\prime \prime}$. Choose $\Gamma^{\prime}=\Gamma, \Psi^{\prime}=\Psi, \overrightarrow{F r}^{\prime}=\overrightarrow{F r}$, and $\sigma=$. Any derivation of 60 has a subderivation of $\Gamma \vdash \vartheta:(\Psi ; \overrightarrow{F r}) \multimap(\Psi ; \overrightarrow{F r} ; \tau)$. Hence $\Gamma \vdash v:\left(\Psi^{\prime} ; \overrightarrow{F r}^{\prime}\right) \multimap\left(\Psi^{\prime \prime} ; \overrightarrow{F r}^{\prime \prime} ; \tau\right)$ holds. Furthermore, $\Gamma^{\prime} \vdash(h ; s):\left(\Psi^{\prime} ; \overrightarrow{F r}^{\prime}\right)$ holds by 59 .

Case 12: $(h, s), \ell v$ stfld $\mu C:: f_{j} \rightsquigarrow\left(h^{\prime}, s\right), 0$ where $h^{\prime}=h\left[\ell \mapsto o b j_{C}\left[f_{j} \mapsto\right.\right.$ $v]]$, and $1 \leq j \leq$ fields $(C)$, and $h(\ell)=o b j_{C}\left\{f_{i}=v_{i} \mid i \in 1 . . f i e l d s(C)\right\} \vec{e}$. By 60 and 41 . $\Gamma^{\prime \prime}=\cdot \Psi=\Psi^{\prime \prime}, \overrightarrow{F r}=\overrightarrow{F r}^{\prime \prime}$, and $\tau=$ void. Choose $\Gamma^{\prime}=\Gamma, \Psi^{\prime}=\Psi, \overrightarrow{F r}^{\prime}=\overrightarrow{F r}$, and $\sigma=\cdot$. Observe that $\Gamma^{\prime} \vdash \mathbf{0}$ : 
$\left(\Psi^{\prime} ; \overrightarrow{F r}^{\prime}\right) \multimap\left(\Psi^{\prime \prime} ; \overrightarrow{F r}^{\prime \prime} ; \tau\right)$ holds by rule 53 . Furthermore, since one derivation of 59 has a subderivation of

$$
\frac{\Gamma \vdash \underline{v_{i}}:(\Psi ; \overrightarrow{F r}) \multimap\left(\Psi ; \overrightarrow{F r} ; \text { field }\left(C, f_{i}\right)\right) \forall i \in 1 . . f i e l d s(C)}{\Gamma \vdash_{\text {heap }} h: \Gamma}
$$

where $\Gamma=\Gamma_{0}, \ell: C$ and $h=h_{0},\left(\ell \mapsto o b j_{C}\{\ldots\}^{\vec{e}}\right)$, and since 60 implies that all three of $\Gamma \vdash v:(\Psi ; \overrightarrow{F r}) \multimap(\Psi ; \overrightarrow{F r} ; \mu)$, field $\left(C, f_{j}\right)=\mu$, and $\ell \in \operatorname{Dom}\left(\Gamma^{\prime}\right)$ hold, one can derive

$$
\begin{gathered}
\Gamma \vdash_{\text {heap }} h_{0}: \Gamma_{0} \\
\Gamma \vdash \vartheta:(\Psi ; \overrightarrow{F r}) \multimap\left(\Psi ; \overrightarrow{F r} ; \text { field }\left(C, f_{j}\right)\right)
\end{gathered}
$$

$\frac{\Gamma \vdash v_{i}:(\Psi ; \overrightarrow{F r}) \multimap\left(\Psi ; \overrightarrow{F r} ; \text { field }\left(C, f_{i}\right)\right) \forall i \in 1 . . j-1, j+1 . . \text { fields }(C)}{\Gamma^{\prime} \vdash_{\text {heap }} h^{\prime}: \Gamma^{\prime}}$

Hence $\Gamma^{\prime} \vdash\left(h^{\prime} ; s\right):\left(\Psi^{\prime} ; \overrightarrow{F r}^{\prime}\right)$ holds.

Case 13: $(h, s), \ell \ell$ evt $e_{1} \rightsquigarrow\left(h^{\prime}, s\right), 0$ where $h^{\prime}=h\left[\ell \mapsto o b j_{C}\{\ldots\}^{\vec{e} e_{1}}\right.$ and $h(\ell)=\operatorname{obj}_{C}\{\ldots\}^{\vec{e}}$. By 60 and $46, \Gamma^{\prime \prime}=\cdot \overrightarrow{F r}=\overrightarrow{F r}^{\prime \prime}, \tau=$ void, $\Psi=\Psi_{1} \star(\ell \mapsto H)$ for some $\Psi_{1}$ and $H$, and $\Psi^{\prime \prime}=\Psi_{1} \star\left(\ell \mapsto H e_{1}\right)$. Choose $\Gamma^{\prime}=\Gamma, \Psi^{\prime}=\Psi^{\prime \prime}, \overrightarrow{F r}^{\prime}=\overrightarrow{F r}$, and $\sigma=\cdot$. Then $\Gamma^{\prime} \vdash \mathbf{0}$ : $\left(\Psi^{\prime} ; \overrightarrow{F r}^{\prime}\right) \multimap\left(\Psi^{\prime \prime} ; \overrightarrow{F r}^{\prime \prime} ; \tau\right)$ holds by typing rule 53 . Furthermore, since one derivation of 59 has a subderivation of

$$
\frac{\vdash_{\text {hist }} h_{0}:\left(\Gamma_{0} ; \Psi_{1}\right) \quad \vec{e} \subseteq H}{\vdash_{\text {hist }} h:(\Gamma ; \Psi)} 228
$$

where $\Gamma=\Gamma_{0}, \ell: C$ and $h=h_{0}, \ell \mapsto o b j_{C}\{\ldots\}^{\vec{e}}$, one can derive

$$
\frac{\vdash_{\text {hist }} h_{0}:\left(\Gamma_{0} ; \Psi_{1}\right) \quad \vec{e} e_{1} \subseteq H e_{1}}{\vdash_{\text {hist }} h^{\prime}:\left(\Gamma ; \Psi^{\prime \prime}\right)}
$$

Hence $\Gamma^{\prime} \vdash\left(h^{\prime} ; s\right):\left(\Psi^{\prime} ; \overrightarrow{F r}^{\prime}\right)$ holds.

Case 14: $(h, s)$, newpackage $C \rightsquigarrow\left(h^{\prime}, s\right), \ell$ where $h^{\prime}=h, \ell \mapsto p k g(\cdot)$. By 60 and $47, \Gamma^{\prime \prime}=\ell: C\langle ?\rangle, \Psi=\Psi^{\prime \prime}, \overrightarrow{F r}=\overrightarrow{F r}^{\prime \prime}$, and $\tau=C\langle ?\rangle$. Choose $\Gamma^{\prime}=\Gamma, \Gamma^{\prime \prime}, \Psi^{\prime}=\Psi, \overrightarrow{F r}^{\prime}=\overrightarrow{F r}$, and $\sigma=\cdot$. Observe that $\Gamma^{\prime} \vdash \ell$ : $\left(\Psi^{\prime \prime} ; \overrightarrow{F r}^{\prime \prime}\right) \multimap\left(\Psi^{\prime \prime} ; \overrightarrow{F r}^{\prime \prime} ; \tau\right)$ by typing rule 56 . Hence $\Gamma^{\prime} \vdash \ell:\left(\Psi^{\prime} ; \overrightarrow{F r}^{\prime}\right) \multimap$ $\exists \Gamma^{\prime \prime} .\left(\Psi^{\prime \prime} ; \overrightarrow{F r}^{\prime \prime} ; \tau\right)$ holds by rule 52 . In addition, any derivation of 59 includes subderivations of $\Gamma \vdash_{\text {heap }} h: \Gamma$ and $\vdash_{\text {hist }} h:(\Gamma ; \Psi)$; hence one can derive

$$
\frac{\Gamma^{\prime} \vdash_{\text {heap }} h: \Gamma}{\Gamma^{\prime} \vdash_{\text {heap }} h^{\prime}: \Gamma^{\prime}}(25) \quad \text { and } \quad \frac{\vdash_{\text {hist }} h:(\Gamma ; \Psi)}{\vdash_{\text {hist }} h^{\prime}:\left(\Gamma^{\prime} ; \Psi^{\prime}\right)}
$$

Thus $\Gamma^{\prime} \vdash\left(h^{\prime} ; s\right):\left(\Psi^{\prime} ; \overrightarrow{F r}^{\prime}\right)$ holds, proving the lemma. 
Case 15: $\ell \ell^{\prime} \quad r e p_{C}(H)$ pack $\rightsquigarrow\left(h^{\prime}, s\right), 0$ where $h(\ell)=p k g(\ldots)$ and $h^{\prime}=$ $h\left[\ell \mapsto p k g\left(\ell^{\prime}, \operatorname{rep}_{C}(H)\right)\right]$. By 60 and $48, \Gamma^{\prime \prime}=\cdot \overrightarrow{F r}=\overrightarrow{F r}^{\prime \prime}, \tau=$ void, and $\Psi=\Psi^{\prime \prime} \star\left(\ell \mapsto H^{\prime}\right)$ for some $H^{\prime}$. Any derivation of 60 has subderivations of $\Gamma \vdash \operatorname{rep}_{C}(H):(\Psi ; \overrightarrow{F r}) \multimap\left(\Psi^{\prime \prime} ; \overrightarrow{F r} ; \operatorname{Rep}_{C}\langle H\rangle\right)$ (by rule 57) such that $H^{\prime} \subseteq H \subseteq$ policy $(C)$ (by rule 48), and of

$$
\frac{\Psi=\Psi^{\prime \prime} \star\left(\ell^{\prime} \mapsto H^{\prime}\right)}{\Gamma \vdash \mid \ell^{\prime}:(\Psi ; \overrightarrow{F r}) \multimap(\Psi ; \overrightarrow{F r} ; C\langle\ell\rangle)}
$$

where $\Gamma=\Gamma_{0}, \ell: C\langle ?\rangle, \ell^{\prime}: C$. One derivation of 59 has a subderivation of

$$
\frac{\mathcal{D}}{\frac{\vdash_{\text {hist }} h_{0},(\ell \mapsto p k g(\ldots)):\left(\Gamma_{0}, \ell: C\langle ?\rangle ; \Psi^{\prime \prime}\right)}{\vdash_{\text {hist }} h:(\Gamma ; \Psi)} \quad \vec{e} \subseteq H^{\prime}}(28)
$$

where $h=h_{0},(\ell \mapsto p k g(\ldots)),\left(\ell^{\prime} \mapsto o b j_{C}\{\ldots\}^{\vec{e}}\right)$ (because rule 28 is the only derivation rule that can add $\ell^{\prime} \mapsto H^{\prime}$ to $\Psi$.) Given the definition of $h_{0}$ above, observe that

$$
h^{\prime}=h_{0},\left(\ell \mapsto p k g\left(\ell^{\prime}, r e p_{C}(H)\right)\right),\left(\ell^{\prime} \mapsto o b j_{C}\{\ldots\}^{\vec{e}}\right)
$$

and $\vec{e} \subseteq H^{\prime} \subseteq H \subseteq$ policy $(C)$. Choose $\Gamma^{\prime}=\Gamma, \Psi^{\prime}=\Psi^{\prime \prime}, \overrightarrow{F r}^{\prime}=\overrightarrow{F r}$, and $\sigma=\cdot$. Observe that $\Gamma^{\prime} \vdash \mathbf{0}:\left(\Psi^{\prime} ; \overrightarrow{F r}^{\prime}\right) \multimap\left(\Psi^{\prime \prime} ; \overrightarrow{F r}^{\prime \prime} ; \tau\right)$ is derivable using rule 53 .

It remains to be shown that $\vdash_{\text {hist }} h^{\prime}:\left(\Gamma^{\prime} ; \Psi^{\prime}\right)$ holds. To prove this, it suffices to prove that $\vdash_{\text {hist }} h_{0}:\left(\Gamma_{0} ; \Psi^{\prime \prime}\right)$ holds, since if this latter judgment holds, one can derive

$$
\frac{\vdash_{h i s t} h_{0}:\left(\Gamma_{0} ; \Psi^{\prime \prime}\right) \quad \vec{e} \subseteq H \subseteq \operatorname{policy}(C)}{\vdash_{\text {hist }} h^{\prime}:\left(\Gamma ; \Psi^{\prime \prime}\right)}
$$

Suppose $h(\ell)=p k g(\cdot)$. Then

$$
\mathcal{D}=\frac{\vdash_{\text {hist }} h_{0}:\left(\Gamma_{0} ; \Psi^{\prime \prime}\right)}{\vdash_{\text {hist }} h_{0},(\ell \mapsto p k g(\cdot)):\left(\Gamma_{0}, \ell: C\langle ?\rangle ; \Psi^{\prime \prime}\right)}
$$

proving that $\vdash_{\text {hist }} h_{0}:\left(\Gamma_{0} ; \Psi^{\prime \prime}\right)$ holds.

Otherwise $h(\ell)=p k g\left(\ell^{\prime \prime}, \operatorname{rep}_{C}\left(H^{\prime \prime}\right)\right)$ for some $\ell^{\prime \prime}$ and $H^{\prime \prime}$. In that case,

$$
\mathcal{D}=\frac{\vdash_{h i s t} h_{1}:\left(\Gamma_{1} ; \Psi^{\prime \prime}\right) \quad \overrightarrow{e^{\prime \prime}} \subseteq H^{\prime \prime} \subseteq \operatorname{policy}(C)}{\vdash_{\text {hist }} h_{0},\left(\ell \mapsto p k g\left(\ell^{\prime \prime}, \operatorname{rep}_{C}\left(H^{\prime \prime}\right)\right)\right):\left(\Gamma_{0}, \ell: C\langle ?\rangle ; \Psi^{\prime \prime}\right)}(29)
$$


where $\Gamma_{0}=\Gamma_{1},\left(\ell^{\prime \prime}: C\right)$ and $\left.h_{0}=h_{1},\left(\ell^{\prime \prime} \mapsto o b j_{C}\{\ldots\}\right\}^{\overrightarrow{e^{\prime \prime}}}\right)$. One can therefore derive

$$
\frac{\vdash_{h i s t} h_{1}:\left(\Gamma_{1} ; \Psi^{\prime \prime}\right) \quad \overrightarrow{e^{\prime \prime}} \subseteq \operatorname{policy}(C)}{\vdash_{\text {hist }} h_{0}:\left(\Gamma_{0} ; \Psi^{\prime \prime}\right)}
$$

proving that $\vdash_{\text {hist }} h_{0}:\left(\Gamma_{0} ; \Psi^{\prime \prime}\right)$ holds.

Case 16: $\left(h, s\left(v_{0}, \ldots, v_{n}\right)\right), \ell$ unpack $j \rightsquigarrow\left(h[\ell \mapsto p k g(\cdot)], s a^{\prime}\right), \sqrt{\ell^{\prime}}$ where $h(\ell)=\operatorname{pkg}\left(\ell^{\prime}, \operatorname{rep}_{C}(H)\right)$ and $a^{\prime}=\left(v_{0}, \ldots, v_{j-1}, \operatorname{rep}_{C}(H), v_{j+1}, \ldots, v_{n}\right)$. By 60 and 51 , $\Gamma^{\prime \prime}=\ell: C, \theta, \Psi^{\prime \prime}=\Psi \star(\ell \mapsto \theta), \tau=C\langle\ell\rangle$, and $\overrightarrow{F r}^{\prime \prime}=$ $\overrightarrow{F r}_{0}\left(\tau_{0}, \ldots, \tau_{j-1}, \operatorname{Rep}_{C}\langle\theta\rangle, \tau_{j+1}, \ldots, \tau_{n}\right)$ where $\overrightarrow{F r}=\overrightarrow{F r}_{0}\left(\tau_{0}, \ldots, \tau_{n}\right)$. One derivation of 59 has a subderivation of

$$
\frac{\vdash_{h i s t} h_{0}:\left(\Gamma_{0} ; \Psi\right) \quad \vec{e} \subseteq H \subseteq \operatorname{policy}(C)}{\vdash_{\text {hist }} h:(\Gamma ; \Psi)}(29)
$$

where $\Gamma=\Gamma_{0}, \ell: C\langle ?\rangle, \ell^{\prime}: C$ and $h=h_{0},\left(\ell \mapsto p k g\left(\ell^{\prime}, \operatorname{rep}_{C}(H)\right)\right),\left(\ell^{\prime} \mapsto\right.$ $\left.\operatorname{obj}_{C}\{\ldots\}^{\vec{e}}\right)$.

Choose $\Gamma^{\prime}=\Gamma, \sigma=(\theta \mapsto \vec{e}), \Psi^{\prime}=\sigma\left(\Psi^{\prime \prime}\right)$, and $\overrightarrow{F r}^{\prime}=\sigma\left(\overrightarrow{F r}^{\prime \prime}\right)$. Since $\theta \notin \operatorname{Dom}(\Gamma)$ (by rule 49$)$, it follows that $\sigma\left(\Psi^{\prime \prime}\right)=\Psi \star(\ell \mapsto \vec{e})$. One can therefore derive

$$
\frac{\Psi^{\prime}=\Psi \star(\ell \mapsto \vec{e})}{\Gamma^{\prime} \vdash \ell^{\prime}:\left(\Psi^{\prime} ; \overrightarrow{F r}^{\prime}\right) \multimap\left(\sigma\left(\Psi^{\prime \prime}\right) ; \sigma\left(\overrightarrow{F r}^{\prime \prime}\right) ; \sigma(\tau)\right)}
$$

and one can derive

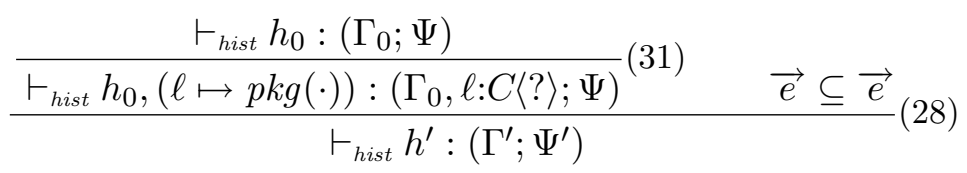

Finally, since any derivation of 59 has a subderivation of

$$
\frac{\Gamma \vdash_{\text {stack }} s: \overrightarrow{F r}_{0} \quad \Gamma \vdash v_{i}:(\Psi ; \overrightarrow{F r}) \multimap\left(\Psi ; \overrightarrow{F r} ; \tau_{i}\right) \forall i \in 0 . . n}{\Gamma \vdash_{\text {stack }} s\left(v_{0}, \ldots, v_{n}\right): \overrightarrow{F r}_{0}\left(\tau_{0}, \ldots, \tau_{n}\right)}
$$

and since $\Gamma \vdash r \operatorname{rep}_{C}(H):\left(\Psi^{\prime} ; \overrightarrow{F r}^{\prime}\right) \multimap\left(\Psi^{\prime} ; \overrightarrow{F r}^{\prime} ; \operatorname{Rep}_{C}\langle H\rangle\right)$ holds by typing rule 57, it follows from derivation rule 34 that $\Gamma^{\prime} \vdash_{\text {stack }} s a^{\prime}: \overrightarrow{F r}^{\prime}$ holds.

Case 17: $\psi, \operatorname{rep}_{C}(H) I_{2} I_{3}$ condst $C, k \rightsquigarrow \psi, I_{j}$ where $j \in\{2,3\}$. Choose $\Gamma^{\prime}=\Gamma, \overrightarrow{F r}^{\prime}=\overrightarrow{F r}$

$$
\Psi^{\prime}= \begin{cases}c t x_{C, k}^{+}(H, \Psi) & \text { if } j=2 \\ c t x_{C, k}^{-}(H, \Psi) & \text { if } j=3\end{cases}
$$


and $\sigma=\cdot$ By 60,51 , and 57 , both $\Gamma \vdash I_{2}:\left(c t x_{C, k}^{+} ; \overrightarrow{F r}\right) \multimap$ $\exists \Gamma^{\prime \prime} .\left(\Psi^{\prime \prime} ; \overrightarrow{F r}^{\prime \prime} ; \tau\right)$ and $\Gamma \vdash I_{3}:\left(c_{x_{C}}^{-} ; \overrightarrow{F r}\right) \multimap \exists \Gamma^{\prime \prime} .\left(\Psi^{\prime \prime} ; \overrightarrow{F r}^{\prime \prime} ; \tau\right)$ hold, so $\Gamma \vdash I_{j}:\left(\Psi^{\prime} ; \overrightarrow{F r}^{\prime}\right) \multimap \exists \Gamma^{\prime \prime} .\left(\Psi^{\prime \prime} ; \overrightarrow{F r}^{\prime \prime} ; \tau\right)$ holds.

Any derivation of 59 has subderivations of $\Gamma \vdash_{\text {heap }} h: \Gamma$ and $\vdash_{\text {hist }}$ $h:(\Gamma ; \Psi)$. To prove that $\Gamma^{\prime} \vdash \psi:\left(\Psi^{\prime} ; \overrightarrow{F r}^{\prime}\right)$, it suffices to show that $\vdash_{\text {hist }} h:\left(\Gamma ; \Psi^{\prime}\right)$. If $j=2$ then $\operatorname{test}_{C, k}\left(\operatorname{rep}_{C}(H)\right) \neq 0$ (by 17), and axiom 20 therefore implies that $\Psi \preceq c t x_{C, k}^{+}(H, \Psi)$. Altnernatively, if $j=3$ then $\operatorname{test}_{C, k}\left(\operatorname{rep}_{C}(H)\right)=0$, and axiom 19 therefore implies that $\Psi \preceq c t x_{C, k}^{-}(H, \Psi)$. In either case, $\Psi \preceq \Psi^{\prime}$ holds. By context subtyping, we conclude that $\vdash_{\text {hist }} h:\left(\Gamma ; \Psi^{\prime}\right)$ also holds.

Case 18: $\psi, v_{1} \ldots v_{n}$ newhist $C, k \rightsquigarrow \psi, h c_{C, k}\left(v_{1}, \ldots, v_{n}\right)$. By 60 and 51 , $\Gamma^{\prime \prime}=\cdot, \Psi=\Psi^{\prime \prime}, \overrightarrow{F r}=\overrightarrow{F r}^{\prime \prime}$, and $\tau=H C_{C, k}\left(\operatorname{Rep}_{C_{1}}\left\langle H_{1}\right\rangle, \ldots, \operatorname{Rep}_{C_{n}}\left\langle H_{n}\right\rangle\right)$ where $\Gamma \vdash v_{i}:(\Psi ; \overrightarrow{F r}) \multimap\left(\Psi ; \overrightarrow{F r} ; \operatorname{Rep}_{C_{i}}\left\langle H_{i}\right\rangle\right)$ holds for all $i \in 1 . . n$. Choose $\Gamma^{\prime}=\Gamma, \Psi^{\prime}=\Psi, \overrightarrow{F r}^{\prime}=\overrightarrow{F r}$, and $\sigma=\cdot$. By axioms 21 and 22 there exists $H$ such that $\tau=\operatorname{Rep}_{C}\langle H\rangle$ and $h c_{C, k}\left(v_{1}, \ldots, v_{n}\right)=$ $\operatorname{rep}_{C}(H)$. Thus, $\Gamma \vdash \operatorname{rep}_{C}(H):\left(\Psi^{\prime} ; \overrightarrow{F r}^{\prime}\right) \multimap\left(\Psi^{\prime \prime} ; \overrightarrow{F r}^{\prime \prime} ; \operatorname{Rep}_{C}\langle H\rangle\right)$ holds by typing rule 57 .

Theorem 3 (Subject Reduction). Assume that $\Gamma \vdash \psi:(\Psi ; \overrightarrow{F r})$ holds and assume that all methods in Dom(methodbody) are well-typed. If $\Gamma \vdash I$ : $(\Psi ; \overrightarrow{F r}) \multimap \exists \Gamma^{\prime \prime} .\left(\Psi^{\prime \prime} ; \overrightarrow{F r}^{\prime \prime} ; \tau\right)$ holds and $\psi, I \rightsquigarrow^{n} \psi^{\prime}, I^{\prime}$ holds then there exist $\Gamma^{\prime}, \Psi^{\prime}, \overrightarrow{F r}^{\prime}$, and $\sigma: \theta \rightarrow \vec{e}$ such that $\Gamma^{\prime} \vdash \psi^{\prime}:\left(\Psi^{\prime} ; \overrightarrow{F r}^{\prime}\right)$ holds and $\Gamma^{\prime} \vdash I^{\prime}$ : $\left(\Psi^{\prime} ; F r^{\prime}\right) \multimap\left(\sigma\left(\Psi^{\prime \prime}\right) ; \sigma\left(F r^{\prime \prime}\right) ; \sigma(\tau)\right)$ holds.

Proof. Proof is by induction on $n$.

Base Case: Assume $n=0$. Choose $\Gamma^{\prime}=\Gamma, \Psi^{\prime}=\Psi, \overrightarrow{F r}^{\prime}=\overrightarrow{F r}$, and $\sigma=\cdot$ The theorem is then satisfied by assumption.

Inductive Case: Assume $n \geq 1$. Since $\psi, I \rightsquigarrow^{n} \psi^{\prime}, I^{\prime}$ holds, there exist $\psi_{1}$ and $I_{1}$ such that $\psi, I \rightsquigarrow^{n-1} \psi_{1}, I_{1}$ holds and such that $\psi_{1}, I_{1} \rightsquigarrow \psi^{\prime}, I^{\prime}$ also holds. By inductive hypothesis, there exists $\Gamma_{1}, \Psi_{1}, \overrightarrow{F r}_{1}$, and $\sigma_{1}$ such that $\Gamma_{1} \vdash \psi_{1}:\left(\Psi_{1} ; \overrightarrow{F r}_{1}\right)$ and $\Gamma_{1} \vdash I_{1}:\left(\Psi_{1} ; \overrightarrow{F r}_{1}\right) \multimap\left(\sigma_{1}\left(\Psi^{\prime \prime}\right) ; \sigma_{1}\left(\overrightarrow{F r}^{\prime \prime}\right) ; \sigma_{1}(\tau)\right)$ hold. The theorem then follows from the stepwise subject reduction lemma. 


\section{B.3 Progress}

Theorem 4 (Progress). Assume $\Gamma \vdash I:(\Psi ; \overrightarrow{F r}) \multimap\left(\Psi^{\prime} ; \overrightarrow{F r}^{\prime} ; \tau\right)$ and $\Gamma \vdash$ $(h ; s):(\Psi ; \overrightarrow{F r})$ hold. Then one of the following conditions holds:

1. $I=v$ for some value $v$.

2. There exists a small-step store $\psi^{\prime}$ and instruction $I^{\prime}$ such that $(h, s), I \rightsquigarrow \psi^{\prime}, I^{\prime}$.

3. $I=E[\ell$ unpack $j]$ and $h(\ell)=p k g(\cdot)$.

Proof. If $I$ is a value, then condition 1 of the theorem holds immediately, proving the theorem. Assume $I$ is not a value. Then $I$ must have one of the following forms:

Case 1: $I=E\left[\mathbf{l d c . i 4} i_{4}\right]$. Condition 2 holds with $I^{\prime}=E\left[i i_{4}\right]$ and $\psi^{\prime}=$ $(h, s)$.

Case 2: $I=E\left[v_{1} ; I_{2}\right]$. Condition 2 holds with $I^{\prime}=E\left[I_{2}\right]$ and $\psi^{\prime}=(h, s)$.

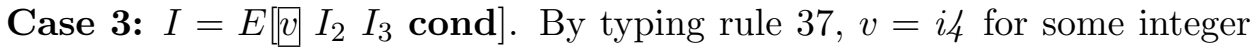
$i 4$. Thus, condition 2 holds with $I^{\prime}=E\left[I_{j}\right]$ and $\psi^{\prime}=(h, s)$, where

$$
j= \begin{cases}3 & \text { if } i_{4}=0 \\ 2 & \text { otherwise }\end{cases}
$$

Case 4: $I=E\left[\begin{array}{lll}I_{1} & I_{2} & \text { while }\end{array}\right]$. Condition 2 holds with

$$
I^{\prime}=E\left[I_{1}\left(I_{2} ;\left(I_{1} I_{2} \text { while }\right)\right) 0 \text { cond }\right]
$$

and $\psi^{\prime}=(h, s)$.

Case 5: $I=E[\operatorname{ldarg} j]$. By typing rule $42, \overrightarrow{F r}=\overrightarrow{F r}_{0}\left(\tau_{0}, \ldots, \tau_{n}\right)$ and $0 \leq j \leq n$. Since $\Gamma \vdash_{\text {stack }} s: \overrightarrow{F r}$, it follows from derivation rule 34 that $s=s_{0}\left(v_{0}, \ldots, v_{n}\right)$. Hence, condition 2 holds with $I^{\prime}=E\left[v_{j}\right]$ and $\psi^{\prime}=(h, s)$.

Case 6: $I=E\left[\overrightarrow{v^{\prime}}\right.$ starg $\left.j\right]$. By typing rule $43, \overrightarrow{F r}=\overrightarrow{F r}_{0}\left(\tau_{0}, \ldots, \tau_{n}\right)$ and $0 \leq j \leq n$. Since $\Gamma \vdash_{\text {stack }} s: \overrightarrow{F r}$, it follows from derivation rule 34 that $s=s_{0}\left(v_{0}, \ldots, v_{n}\right)$. Hence, condition 2 holds with $I^{\prime}=E[\mathbf{0}]$ and $\psi^{\prime}=\left(h, s_{0}\left(v_{0}, \ldots, v_{j-1}, v^{\prime}, v_{j+1}, \ldots, v_{n}\right)\right)$.

Case 7: $I=E\left[v_{1} \ldots v_{n}\right.$ newobj $\left.C\left(\mu_{1}, \ldots, \mu_{n}\right)\right]$. Typing rule 44 implies that $n=$ fields $(C)$. Condition therefore 2 holds with $I^{\prime}=E[\ell]$ and $\psi^{\prime}=\left(h\left[\ell \mapsto o b j_{C}\left\{f_{i} \mapsto v_{i} \mid i \in 1 . . n\right\}^{\epsilon}\right], s\right)$. 
Case 8: $I=E\left[v_{0} \ldots v_{n}\right.$ callvirt $\left.C:: m . S i g\right]$. By typing rule 45 there exists $I_{0}$ such that methodbody $(C:: m . S i g)=I_{0}$. Hence, condition 2 holds with $I^{\prime}=E\left[I_{0}\right.$ ret $]$ and $\psi^{\prime}=\left(h, s\left(v_{0}, \ldots, v_{n}\right)\right)$.

Case 9: $I=E[v$ ldfld $\mu C:: f]$. By typing rule 40, $v=\ell$ such that $\Gamma \vdash$ $\ell:(\Psi ; \overrightarrow{F r}) \multimap(\Psi ; \overrightarrow{F r} ; C\langle\ell\rangle)$ holds. Since $\Gamma \vdash_{\text {heap }} h: \Gamma$ holds, it follows from derivation rule 24 that $h(\ell)=o b j_{C}\{\ldots, f=v, \ldots\} \vec{e}$ for some value $v$. Hence, condition 2 holds with $I=E[v]$ and $\psi^{\prime}=(h, s)$.

Case 10: $I=E[v \sqrt{v}$ stfld $\mu C:: f]$. By typing rule $41, v=\ell$ such that $\Gamma \vdash \ell:(\Psi ; \overrightarrow{F r}) \multimap(\Psi ; \overrightarrow{F r} ; C\langle\ell\rangle)$ holds. Since $\Gamma \vdash_{\text {heap }} h: \Gamma$ holds, it follows from derivation rule 24 that $h(\ell)=o b j_{C}\{\ldots, f=v, \ldots\} \vec{e}$ for some value $v$. Hence, condition 2 holds with $I=E[\mathbf{0}]$ and $\psi^{\prime}=(h[\ell \mapsto$ $\left.\left.\operatorname{obj}_{C}\left[f \mapsto v^{\prime}\right]\right], s\right)$.

Case 11: $I=E\left[v\right.$ evt $\left.e_{1}\right]$. By typing rule $46, v=\ell$ such that $\Gamma \vdash \ell$ : $(\Psi ; \overrightarrow{F r}) \multimap(\Psi ; \overrightarrow{F r} ; C\langle\ell\rangle)$ holds. Since $\Gamma \vdash_{\text {heap }} h: \Gamma$ holds, it follows from derivation rule 24 that $h(\ell)=o b j_{C}\{\ldots\}^{\vec{e}}$ for some event sequence $\vec{e}$. Thus, condition 2 of the theorem holds with $I^{\prime}=E[\mathbf{0}]$ and $\psi^{\prime}=(h[\ell \mapsto$ $\left.o b j_{C}\{\ldots\}^{\vec{e}} e_{1}, s\right)$.

Case 12: $I=E[$ newpackage $C]$. Choose $\ell \notin D o m(h)$. Condition 2 holds with $I^{\prime}=E[\ell]$ and $\psi^{\prime}=((h,(\ell \mapsto p k g(\cdot))), s)$.

Case 13: $I=E\left[v v^{\prime} v^{\prime \prime}\right.$ pack]. By typing rule $46, v=\ell$ such that $\Gamma \vdash$ $\ell:(\Psi ; \overrightarrow{F r}) \multimap(\Psi ; \overrightarrow{F r} ; C\langle ?\rangle)$ holds, $v^{\prime}=\ell^{\prime}$ for some heap pointer $\ell^{\prime}$, and $v^{\prime \prime}=\operatorname{rep}_{C}(H)$ for some history abstraction $H$. Since $\Gamma \vdash_{\text {heap }} h: \Gamma$ holds, it follows from derivation rule 25 that $h(\ell)=p k g(\ldots)$. Hence, condition 2 of the theorem holds with $I^{\prime}=E[\mathbf{0}]$ and $\psi^{\prime}=(h[\ell \mapsto$ $\left.\left.\operatorname{pkg}\left(\ell^{\prime}, \operatorname{rep}_{C}(H)\right)\right], s\right)$.

Case 14: $I=E[v$ unpack $j]$. By typing rule 49, $v=\ell$ such that $\Gamma \vdash$ $\ell:(\Psi ; \overrightarrow{F r}) \multimap(\Psi ; \overrightarrow{F r} ; C\langle ?\rangle)$ holds, and $\overrightarrow{F r}=\overrightarrow{F r}_{0}\left(\tau_{0}, \ldots, \tau_{n}\right)$ where $0 \leq j \leq n$. Since $\Gamma \vdash_{\text {stack }} s: \overrightarrow{F r}$, it follows from derivation rule 34 that $s=s_{0}\left(v_{0}, \ldots, v_{n}\right)$. Since $\Gamma \vdash_{\text {heap }} h: \Gamma$, it follows from derivation rule 25 that $h(\ell)=p k g(\ldots)$. If $h(\ell)=p k g\left(\ell^{\prime}, v\right)$, then condition 2 of the theorem holds with $I=E\left[\ell^{\prime}\right]$ and $\psi^{\prime}=(h[\ell \mapsto$ $\left.p k g(\cdot)], s_{0}\left(v_{0}, \ldots, v_{j-1}, v, v_{j+1}, \ldots, v_{n}\right)\right)$. Otherwise $h(\ell)=p k g(\cdot)$ and therefore condition 3 of the theorem holds.

Case 15: $I=E[v] I_{2} I_{3}$ condst $\left.k\right]$. By typing rule [50, $v=\operatorname{rep}_{C}(H)$ for some class $C$ and history abstraction $H$. Condition 2 therefore holds with

$$
I^{\prime}= \begin{cases}E\left[I_{3}\right] & \text { if } \operatorname{test}_{k}\left(C, \operatorname{rep}_{C}(H)\right)=0 \\ E\left[I_{2}\right] & \text { otherwise }\end{cases}
$$


and $\psi^{\prime}=(h, s)$.

Case 16: $I=E\left[v_{1} \ldots v_{n}\right.$ newhist $\left.k\right]$. By typing rule 51, $\operatorname{arity}\left(H C_{k}\right)=n$. By axiom 22, it therefore follows that $\operatorname{arity}\left(h c_{k}\right)=n$. Condition 2 of the theorem statement therefore holds with $I^{\prime}=E\left[h c_{k}\left(v_{1}, \ldots, v_{n}\right)\right.$ and $\psi^{\prime}=(h, s)$.

\section{B.4 Policy Adherence}

The proof of Terminating Policy Adherence (Theorem 1) is as follows.

Proof. By subject reduction, there exists $\Gamma^{\prime}, \Psi^{\prime}, \overrightarrow{F r}^{\prime}$, and $\sigma$ such that $\Gamma^{\prime} \vdash$ $v:\left(\Psi^{\prime} ; F r^{\prime}\right) \multimap\left(\sigma\left(\Psi_{\text {out }}\right) ; \sigma\left(F r_{\text {out }}\right) ; \sigma\left(\tau_{\text {out }}\right)\right)$ and $\Gamma^{\prime} \vdash\left(h^{\prime} ; s^{\prime}\right):\left(\Psi^{\prime} ; F r^{\prime}\right)$ hold. From the typing rules for value expressions, we know that $\Psi^{\prime}=\sigma\left(\Psi_{\text {out }}\right)$ and $F r^{\prime}=\sigma\left(F r_{\text {out }}\right)$. Thus

$$
\Gamma^{\prime} \vdash\left(h^{\prime} ; s^{\prime}\right):\left(\sigma\left(\Psi_{\text {out }}\right) ; \sigma\left(F r_{\text {out }}\right) ; \sigma(\vartheta)\right)
$$

holds.

Let $\ell$ and $\vec{e}$ be given such that $h^{\prime}(\ell)=o b j_{C}\{\ldots\} \vec{e}$. If $\ell \in \operatorname{Dom}\left(\Psi_{\text {out }}\right)$ then there exists a derivation of 62 with a subderivation of

$$
\frac{\vdots \quad \vec{e} \subseteq \sigma\left(\Psi_{\text {out }}(\ell)\right)}{\vdash_{\text {hist }} h^{\prime}:\left(\Gamma^{\prime} ; \Psi^{\prime}\right)}
$$

Since $\sigma\left(\Psi_{\text {out }}(\ell)\right) \subseteq$ policy $(C)$ by assumption, we conclude that $\vec{e} \subseteq$ $\operatorname{policy}(C)$. If instead $\ell \notin \operatorname{Dom}\left(\Psi_{\text {out }}\right)$, then there exists a derivation of 62 with either a subderivation of

$$
\frac{\vdots \quad \vec{e} \subseteq \cdots \subseteq \operatorname{policy}(C)}{\vdash_{h i s t} h^{\prime}:\left(\Gamma^{\prime} ; \Psi^{\prime}\right)} 29
$$

or a subderivation of

$$
\frac{\vdots \quad \vec{e} \subseteq \operatorname{policy}(C)}{\vdash_{\text {hist }} h^{\prime}:\left(\Gamma^{\prime} ; \Psi^{\prime}\right)}
$$

In either case, we conclude that $\vec{e} \subseteq$ policy $(C)$, satsifying the theorem.

The proof of Non-terminating Prefix Adherence (Theorem 2 is as follows.

Proof. Proof is by induction on $n$. 
Base Case: If $n=0$ then $h^{\prime}=h$ and the theorem holds by assumption.

Inductive Case: If $n \geq 1$ then there exists $h_{1}, s_{1}$, and $I_{1}$ such that $(h, s), I \rightsquigarrow^{n-1}\left(h_{1}, s_{1}\right), I_{1}$ holds and $\left(h_{1}, s_{1}\right), I_{1} \rightsquigarrow\left(h^{\prime}, s^{\prime}\right), I^{\prime}$ holds. By inductive hypothesis, $h_{1}$ is prefix-adherent. By subject reduction, there also exists $\Gamma_{1}, \Psi_{1}, F r_{1}$, and $\sigma$ such that $\Gamma_{1} \vdash I_{1}:\left(\Psi_{1} ; F r_{1}\right) \multimap$ $\left(\sigma\left(\Psi^{\prime}\right) ; \sigma\left(\overrightarrow{F r}^{\prime}\right) ; \sigma(\tau)\right)$ and $\Gamma_{1} \vdash\left(h_{1} ; s_{1}\right):\left(\Psi_{1} ; \overrightarrow{F r}_{1}\right)$ hold.

Suppose $I_{1}=E\left[v_{1} \ldots v_{n}\right.$ newobj $\left.C\left(\mu_{1}, \ldots, \mu_{m}\right)\right]$. Then $h^{\prime}=h,(\ell \mapsto$ $\left.\operatorname{obj}_{C}\{\ldots\}^{\epsilon}\right)$. Typing rule 44 implies that $\epsilon \in \operatorname{pre}(\operatorname{policy}(C))$. Since $h$ is prefix-adherent, we conclude that $h^{\prime}$ is also prefix-adherent.

Suppose $I_{1}=E\left[\ell\right.$ evt $\left.e_{1}\right]$. Then $h$ and $h^{\prime}$ are identical except for the event history of class object $h(\ell)=o b j_{C}\{\ldots\}^{\vec{e}}$. Typing rule 46 implies that $\Psi_{1}(\ell) e_{1} \subseteq$ pre $($ policy $(C))$. Since $\ell \in \operatorname{Dom}\left(\Psi_{1}\right)$ there exists a derivation of $\Gamma_{1} \vdash\left(h_{1} ; s_{1}\right):\left(\Psi_{1} ; \overrightarrow{F r}_{1}\right)$ that includes a subderivation of

$$
\frac{\vdots \quad \vec{e} \subseteq \Psi_{1}(\ell)}{\vdash_{\text {hist }}\left(h_{1} ; s_{1}\right):\left(\Gamma_{1} ; \Psi_{1}\right)}
$$

Thus, $\vec{e} e_{1} \subseteq \operatorname{pre}(\operatorname{policy}(C))$, and we conclude that $h^{\prime}$ is prefixadherent.

If $I_{1}$ has any other form, then $h$ and $h^{\prime}$ are identical with respect to the event histories of their class objects. Since $h$ is prefix-adherent by assumption, it follows that $h^{\prime}$ is prefix-adherent.

\section{Deciding Subset Relations}

The typing rules presented in Appendix A require a type-checker to decide subset relations over the language of history abstractions given in Figure 3 . History abstractions are $\omega$-regular expressions with variables and intersection. In general, deciding subset for such a language is intractable, but not every history abstraction expression can appear in practice. Our implementation of Mobile decides subset for a sub-language of the language of history abstractions. We present this sub-language below, we argue that it captures most of the useful history abstractions that can appear in practice, and we prove that subset over this language can be reduced to subset over the language of regular expressions.

\section{C.1 History Variables and Intersection}

Intersection is introduced into a history abstraction during type-checking by typing rule 50 (the typing rule for condst). In our implementation, this 
typing rule substitutes an expression of the form $\theta \cap H$ for each occurrence of variable $\theta$, where $H$ is a closed history abstraction. Since intersection is introduced in no other way, this reduces the language of history abstractions of interest to the following sub-language of the language given in Figure 3 ;

$$
\begin{aligned}
& H::=\epsilon|e| H_{1} H_{2}\left|H_{1} \cup H_{2}\right| H^{\omega} \mid V \\
& V::=\theta \mid V \cap C \\
& C::=\epsilon|e| C_{1} C_{2}\left|C_{1} \cup C_{2}\right| C^{\omega}
\end{aligned}
$$

Since our history abstractions are intended to model security automata, each closed history abstraction $C$ introduced by the condst typing rule denotes the set of traces that can cause the automaton to enter a particular state. Since the automata are deterministic, for any pair $C_{1}, C_{2}$ of these abstractions, either $C_{1}=C_{2}$ or $C_{1} \cap C_{2}=\emptyset$. Thus, we can conservatively approximate a history abstraction of the form $\theta \cap C_{1} \cap C_{2}$ with an abstraction of the form $\theta \cap C_{1}$. The latter is guaranteed to be a superset of the former, and no IRM that models security policies using deterministic security automata will be affected by the conservative approximation 3 This further reduces the language of history abstractions to

$$
\begin{aligned}
& H::=\epsilon|e| H_{1} H_{2}\left|H_{1} \cup H_{2}\right| H^{\omega} \mid V \\
& V::=\theta \cap C \\
& C::=\epsilon|e| C_{1} C_{2}\left|C_{1} \cup C_{2}\right| C^{\omega}
\end{aligned}
$$

History variables are further constrained in where they can appear. No typing rule allows an open history abstraction to be appended to a closed history abstraction. History variables introduced in conditional branches and in loops are required to alpha-vary at join points for those conditionals and loops so that there is only ever one unique history variable per history abstraction. (This ensures that there are only a finite number of history variables in scope at any given control flow point.) Our implementation therefore only supports history abstractions of the form

$$
\begin{aligned}
& H::=\left(\theta \cap C_{1}\right) C_{2} \mid C \\
& C::=\epsilon|e| C_{1} C_{2}\left|C_{1} \cup C_{2}\right| C^{\omega}
\end{aligned}
$$

\section{C.2 Reduction to Regular Expression Subset}

In this section we reduce the subset relation for the above language to subset over regular expressions.

\footnotetext{
${ }^{3}$ IRM's that do not model security policies using deterministic automata will be affected in that they will not be able to usefully "stack" dynamic state tests. That is, doing a second state test within a conditional branch of another state test will not cause the type-checker to infer the conjunction of the two tests; rather, the type-checker will conservatively infer typing refinements from only one of the tests, ignoring the other.
} 
Subset problems for the above language can appear in one of five possible forms:

1. $\forall \theta \cdot\left(\left(\theta \cap C_{1}\right) C_{2} \subseteq\left(\theta \cap C_{1}^{\prime}\right) C_{2}^{\prime}\right)$,

2. $\forall \theta_{1}, \theta_{2} \cdot\left(\left(\theta_{1} \cap C_{1}\right) C_{2} \subseteq\left(\theta_{2} \cap C_{1}^{\prime}\right) C_{2}^{\prime}\right)$ (where $\left.\theta_{1} \neq \theta_{2}\right)$,

3. $\forall \theta \cdot\left(\left(\theta \cap C_{1}\right) C_{2} \subseteq C\right)$,

4. $\forall \theta .\left(C \subseteq\left(\theta \cap C_{1}\right) C_{2}\right)$, or

5. $C \subseteq C^{\prime}$.

That is, there is either one history variable on both sides of the subset problem (1), a different history variable on each side (2), a single history variable on one side but none on the other (3 and 4), or no history variables at all (5). In Theorems 5 , we show that each of the first four forms can be reduced to the fifth form. Then in Theorem 9 we reduce the fifth form to the subset problem for regular expressions.

Lemma 4. Every closed, non-empty $\omega$-regular expression has a finite-length member.

Proof. Proof is by induction on the structure of the $\omega$-regular expression $H$. If $H$ has the form $\epsilon$ or $e$, then the lemma follows immediately. If $H$ has the form $H_{1} H_{2}, H_{1} \cup H_{2}$, or $H_{1} \cap H_{2}$, then the lemma follows from inductive hypothesis. If $H$ has the form $H_{1}^{\omega}$, then the lemma holds because $\epsilon \in H_{1}^{\omega}$.

Theorem 5. The following two statements are equivalent:

(i) $\forall \theta \cdot\left(\left(\theta \cap C_{1}\right) C_{2} \subseteq\left(\theta \cap C_{1}^{\prime}\right) C_{2}^{\prime}\right)$

(ii) $\left(C_{1} \subseteq \emptyset\right) \vee\left(C_{2} \subseteq \emptyset\right) \vee\left(\left(C_{1} \subseteq C_{1}^{\prime}\right) \wedge\left(C_{2} \subseteq C_{2}^{\prime}\right)\right)$

Proof. We begin by proving that (ii) implies (i). If $C_{1} \subseteq \emptyset$ or $C_{2} \subseteq \emptyset$ holds then $\left(\theta \cap C_{1}\right) C_{2}=\emptyset$ holds and the theorem is proved. Assume instead that $C_{1}$ and $C_{2}$ are both non-empty, and that $C_{1} \subseteq C_{1}^{\prime}$ and $C_{2} \subseteq C_{2}^{\prime}$ hold. Then $\left(\theta \cap C_{1}\right) \subseteq\left(\theta \cap C_{1}^{\prime}\right)$ holds, and hence $\left(\theta \cap C_{1}\right) C_{2} \subseteq\left(\theta \cap C_{1}^{\prime}\right) C_{2}^{\prime}$ holds.

It remains to show that (i) implies (ii). Assume that for all sets $\theta$, $\left(\theta \cap C_{1}\right) C_{2} \subseteq\left(\theta \cap C_{1}^{\prime}\right) C_{2}^{\prime}$ holds. If $C_{1} \subseteq \emptyset$ or $C_{2} \subseteq \emptyset$ then the theorem follows immediately, so assume that $C_{1}$ and $C_{2}$ are both non-empty. First, we prove that $C_{1} \subseteq C_{1}^{\prime}$ holds. Instantiate $\theta=C_{1} \backslash C_{1}^{\prime}$ (where $\backslash$ denotes set difference). Then $\left(C_{1} \backslash C_{1}^{\prime}\right) C_{2} \subseteq \emptyset$ holds. Since $C_{2}$ is non-empty by assumption, it follows that $C_{1} \backslash C_{1}^{\prime}=\emptyset$ holds, and therefore $C_{1} \subseteq C_{1}^{\prime}$ holds. Second, we prove that $C_{2} \subseteq C_{2}^{\prime}$ holds. Since $C_{1}$ is non-empty, Lemma 4 guarantees that there exists a finite member $s \in C_{1}$. Instantiate $\theta=s$. Then $s C_{2} \subseteq\left(s \cap C_{1}^{\prime}\right) C_{2}^{\prime}$ holds. Since $C_{2}$ is non-empty, $s C_{2}$ is also non-empty, and therefore $\left(s \cap C_{1}^{\prime}\right)$ and 
$C_{2}^{\prime}$ are non-empty. Since $\left(s \cap C_{1}^{\prime}\right)$ is non-empty, it follows that $\left(s \cap C_{1}^{\prime}\right)=s$. Therefore $s C_{2} \subseteq s C_{2}^{\prime}$ holds, and we conclude that $C_{2} \subseteq C_{2}^{\prime}$ holds.

Theorem 6. The following two statements are equivalent:

(i) $\forall \theta_{1}, \theta_{2} \cdot\left(\left(\theta_{1} \cap C_{1}\right) C_{2} \subseteq\left(\theta_{2} \cap C_{1}^{\prime}\right) C_{2}^{\prime}\right)$ (where $\left.\theta_{1} \neq \theta_{2}\right)$

(ii) $\left(C_{1} \subseteq \emptyset\right) \vee\left(C_{2} \subseteq \emptyset\right)$

Proof. To prove that (i) implies (ii), assume that for all sets $\theta_{1}$ and $\theta_{2}$, $\left(\theta_{1} \cap C_{1}\right) C_{2} \subseteq\left(\theta_{2} \cap C_{1}^{\prime}\right) C_{2}^{\prime}$ holds. Instantiate $\theta_{1}=C_{1}$ and $\theta_{2}=\emptyset$. It follows that $C_{1} C_{2} \subseteq \emptyset$ holds, and therefore $C_{1} \subseteq \emptyset$ or $C_{2} \subseteq \emptyset$ hold.

To prove that (ii) implies (i), assume instead that $C_{1} \subseteq \emptyset$ or $C_{2} \subseteq \emptyset$ hold. Then $\left(\theta \cap C_{1}\right) C_{2}=\emptyset$ holds, and the theorem follows immediately.

Theorem 7. The following two statements are equivalent:

(i) $\forall \theta \cdot\left(\left(\theta \cap C_{1}\right) C_{2} \subseteq C\right)$

(ii) $C_{1} C_{2} \subseteq C$

Proof. To prove that (i) implies (ii), assume that for all sets $\theta,\left(\theta \cap C_{1}\right) C_{2} \subseteq C$ holds. Instantiate $\theta=C_{1}$ and it follows that $C_{1} C_{2} \subseteq C$ holds.

To prove that (ii) implies (i), assume instead that $C_{1} C_{2} \subseteq C$ holds. Then for all sets $\theta,\left(\theta \cap C_{1}\right) C_{2} \subseteq C_{1} C_{2} \subseteq C$ holds, proving the theorem.

Theorem 8. The following two statements are equivalent:

(i) $\forall \theta \cdot\left(C \subseteq\left(\theta \cap C_{1}\right) C_{2}\right)$

(ii) $C \subseteq \emptyset$

Proof. To prove that (i) implies (ii), instantiate $\theta=\emptyset$ in (i) and (ii) follows immediately. To prove that (ii) implies (i), substitute $\emptyset$ for $C$ in (i).

The above four proofs demonstrate that subset problems involving variables and intersection can all be reduced to four or fewer instances of subset problems over closed $\omega$-regular expressions. We now show that the subset problem for $\omega$-regular expressions without Kleene star can be reduced to the subset problem for regular expressions.

Lemma 5. Let $C$ be an $\omega$-regular expression without Kleene star, and define $R$ to be the same expression but with all $\omega$ 's replaced with Kleene stars. The set denoted by $R$ is the set of finite-length members of the set denoted by $C$. 
Proof. Proof is by induction on the structure of expression $C$.

If $C=\emptyset, C=\epsilon$, or $C=e$, then $C$ contains only finite-length sequences and $C=R$.

If $C=C_{1} C_{2}$ or $C=C_{1} \cup C_{2}$, then $R=R_{1} R_{2}$ or $R=R_{1} \cup R_{2}$ (respectively), where $R_{1}$ and $R_{2}$ are $C_{1}$ and $C_{2}$ (respectively) with any $\omega$ 's replaced by Kleene stars. By inductive hypothesis, $R_{1}$ is the set of finite members of $C_{1}$ and $R_{2}$ is the set of finite members of $C_{2}$. It follows that $R_{1} R_{2}$ is the set of finite members in $C_{1} C_{2}$ and $R_{1} \cup R_{2}$ is the set of finite members in $C_{1} \cup C_{2}$.

If $C=C_{1}^{\omega}$ then $R=R_{1}^{*}$ where $R_{1}$ is $C_{1}$ with any $\omega$ 's replaced by Kleene stars. By inductive hypothesis, $R_{1}$ is the set of finite-length members of $C_{1}$; therefore $R_{1}^{*}$ is the set of finite-length members of $C_{1}^{\omega}$.

Lemma 6. Let $C$ be an $\omega$-regular expression without Kleene star. For every infinite-length sequence $s \in C$, and for all integers $i \geq 0$, there exists a finite sequence $s^{\prime} \in C$ such that $s$ and $s^{\prime}$ have identical length-i prefixes.

Proof. Proof is by induction on the structure of $C$. Let $s \in C$ and $i \geq 0$ be given, and assume that $s$ is infinite.

If $C=\emptyset, C=\epsilon$, or $C=e$, then $C$ contains only finite-length sequences and the lemma holds vacuously.

If $C=C_{1} C_{2}$ then $s \in C_{1}$ or $s=s_{1} s_{2}$ where $s_{1} \in C_{1}$ is finite and $s_{2} \in C_{2}$ is infinite. If $s \in C_{1}$ then the lemma holds immediately by inductive hypothesis. Otherwise since $s_{2} \in C_{2}$, by inductive hypothesis there is a finite sequence $s_{3} \in C_{2}$ such that $s_{2}$ and $s_{3}$ have identical length- $i$ prefixes. Hence, $s_{1} s_{3} \in C$ is finite and has a length- $i$ prefix identical to that of $s$.

If $C=C_{1} \cup C_{2}$ then $s \in C_{1}$ or $s \in C_{2}$. By inductive hypothesis, there exists a finite sequence $s^{\prime} \in C_{1}$ or $s^{\prime} \in C_{2}$ such that $s$ and $s^{\prime}$ have identical length- $i$ prefixes.

Finally, if $C=C_{1}^{\omega}$ then $s=s_{1} s_{2} s_{3} \cdots$ such that for all $j \geq 1, s_{j} \in C_{1}$. That is, $s$ is a concatenation of a finite or infinite collection of sequences drawn from $C_{1}$. Since $s$ is infinite, one can choose an integer $k \geq 1$ such that $s^{\prime}=s_{1} s_{2} \cdots s_{k}$ has length at least $i$. Without loss of generality, assume that $k$ is the smallest such integer. Observe that $s$ and $s^{\prime}$ have identical length- $i$ prefixes and that $s^{\prime} \in C$ (because $s^{\prime}$ is a concatenation of a finite collection of sequences from $C_{1}$ ). If $s^{\prime}$ is finite then the lemma is satisfied. Assume instead that $s^{\prime}$ is infinite. Then $s_{k}$ is infinite. Since $s_{k} \in C_{1}$ is infinite, by inductive hypothesis there exists $s^{\prime \prime} \in C_{1}$ such that $s^{\prime \prime}$ is finite and $s_{k}$ and $s^{\prime \prime}$ have identical length- $i$ prefixes. Hence, $s_{1} \cdots s_{k-1} s^{\prime \prime} \in C$ is finite and has a length- $i$ prefix identical to that of $s$.

Theorem 9. Let $C_{1}$ and $C_{2}$ be closed $\omega$-regular expressions without Kleene star, and define $R_{1}$ and $R_{2}$ to be the same expressions but with all $\omega$ 's replaced by Kleene stars. Then $C_{1} \subseteq C_{2}$ if and only if $R_{1} \subseteq R_{2}$. 
Proof. We first prove the forward implication. Assume that $C_{1} \subseteq C_{2}$. Then the set of finite-length sequences in $C_{1}$ is a subset of the set of finite-length sequences in $C_{2}$. By Lemma 5, $R_{1}$ is the set of finite-length sequences in $C_{1}$ and $R_{2}$ is the set of finite-length sequences in $C_{2}$, so we conclude that $R_{1} \subseteq R_{2}$.

We next prove the inverse of the forward implication. Assume there exists $s \in C_{1}$ such that $s \notin C_{2}$. If $s$ is finite then by Lemma $5, s \in R_{1}$ and $s \notin R_{2}$, and therefore $R_{1} \nsubseteq R_{2}$, proving the theorem.

Assume instead that $s$ is infinite. Since $s \notin C_{2}$, there exists $i \geq 1$ such that no member of $C_{2}$ has a length- $i$ prefix matching that of $s$. Lemma 6 implies that there exists a finite sequence $s^{\prime} \in C_{1}$ such that $s$ and $s^{\prime}$ have matching length- $i$ prefixes. Since $s^{\prime} \in C_{1}$ is finite, Lemma 5 implies that $s^{\prime} \in R_{1}$. However, since $C_{2}$ has no members with length- $i$ prefixes that match that of $s^{\prime}$, Lemma 5 also implies that there are no members of $R_{2}$ with length- $i$ prefixes that match that of $s^{\prime}$. Hence $s^{\prime} \notin R_{2}$, and we conclude that $R_{1} \nsubseteq R_{2}$, proving the theorem.

The theorems presented in this subsection yield a simple algorithm for deciding subset over the sub-language of history abstractions defined in the previous subsection. That is, Theorems 58 reduce the subset problem for history abstractions with variables and intersection to four or fewer instances of the subset problem for history abstractions without variables or intersection. Then Theorem 9 shows that subset for history abstractions without variables or intersection can be computed by changing all $\omega$ 's into Kleene stars and deciding subset for the resulting regular expressions. 